University of Louisville

ThinkIR: The University of Louisville's Institutional Repository

Electronic Theses and Dissertations

$5-2013$

\title{
Cigarette smoke promotes genomic evolution in the periodontal pathogen Porphyromonas gingivalis.
}

Josh George

University of Louisville

Follow this and additional works at: https://ir.library.louisville.edu/etd

\section{Recommended Citation}

George, Josh, "Cigarette smoke promotes genomic evolution in the periodontal pathogen Porphyromonas gingivalis." (2013). Electronic Theses and Dissertations. Paper 486.

https://doi.org/10.18297/etd/486

This Master's Thesis is brought to you for free and open access by ThinkIR: The University of Louisville's Institutional Repository. It has been accepted for inclusion in Electronic Theses and Dissertations by an authorized administrator of ThinkIR: The University of Louisville's Institutional Repository. This title appears here courtesy of the author, who has retained all other copyrights. For more information, please contact thinkir@louisville.edu. 


\title{
CIGARETTE SMOKE PROMOTES GENOMIC EVOLUTION IN THE PERIODONTAL PATHOGEN
}

\section{PORPHYROMONAS GINGIVALIS}

\author{
by \\ JOSH GEORGE \\ B.S. Biology \\ A Thesis \\ Submitted to the Faculty of the \\ School of Dentistry of the University of Louisville \\ In Partial Fulfillment of the Requirements \\ For the Degree of \\ Master of Science \\ Oral Biology \\ University of Louisville \\ Louisville, Kentucky
}

May 2013 



\section{CIGARETTE SMOKE PROMOTES GENOMIC EVOLUTION \\ IN THE PERIODONTAL PATHOGEN \\ PORPHYROMONAS GINGIVALIS}

by

JOSH GEORGE

B.S. Biology

A Thesis Approved on

April 26, 2013

By the following Thesis Committee:

David Scott Ph.D. - Mentor/Director

Ted Kalbfleisch Ph.D. - Committee Member

Jan Potempa Ph.D. - Committee Member

Don Demuth Ph.D. - Committee Member 


\section{ACKNOWLEDGMENTS}

I would like to thank Dr. David Scott for taking me on as a Master's student and mentoring me during my time here. It has been a privilege to work with such an accomplished investigator. Your guidance and patience over the last few years has been greatly appreciated. I know the experience and knowledge I have gained from this research will serve in making me a better dental clinician in the future.

I would also like to thank Dr. Iris Zeller for allowing me the opportunity to work with her on the $P$. gingivalis genome project, as well as her guidance throughout. Without your help Iris, none of this would have been possible.

Ted Kalbfleisch and Guy Brock have also been indispensible for this project. Many thanks to you both. Ted, for all your bioinformatics work and the answers to my numerous questions and emails, as well as serving on my thesis committee. Guy, for all your work statistically analyzing the enormous amounts of data.

I would also like to give special thanks to the other members of Scott lab - Diane Renaud and Justin Hutcherson for all the time you have given me over the last two years teaching me protocols, guiding me, and answering my numerous questions.

Lastly, I would like to acknowledge Dr. Don Demuth and Dr. Jan Potempa for serving on my thesis committee. 


\author{
ABSTRACT \\ CIGARETTE SMOKE PROMOTES GENOMIC EVOLUTION \\ IN THE PERIODONTAL PATHOGEN \\ PORPHYROMONAS GINGIVALIS \\ Josh George
}

$04 / 26 / 2013$

P. gingivalis is an opportunistic, anaerobic, Gram-negative oral pathogen strongly implicated in the pathogenesis of periodontal diseases. Clinical isolates of $P$. gingivalis exhibit great genetic diversity. Tobacco smoking greatly increases the risk of $P$. gingivalis infection. Furthermore, cigarette smoke extract (CSE) is an environmental stressor for $P$. gingivalis, differentially regulating multiple genes including ones that encode proteins involved in DNA translocation, replication and repair. Therefore, we hypothesized that CSE exposure would promote genomic instability in this important periodontal and systemic pathogen, which may contribute to increased virulence.

P. gingivalis W83 was passaged 50 times in CSE-conditioned medium after which, genomic DNA was extracted and whole-genome sequencing was employed to identify any chromosomal alterations induced by CSE compared to un-exposed controls. 
Whole genome sequencing identified 22 CSE-induced point mutations $(\mathrm{p}<0.05)$, 17 or which were confirmed as well as significant allelic variance $(p<0.001)$ in CSE versus control samples. Our results indicate that CSE exposure promotes genetic diversity in a laboratory strain of $P$. gingivalis. Should this phenomenon occur in vivo, tobacco smoke could promote bacterial evolution and, thus, contribute to the emergence of more virulent strains in this key periodontal pathogen, which may infect smokers and non-smokers alike. 


\section{TABLE OF CONTENTS}

ACKNOWLEDGEMENTS .................................................................... iii

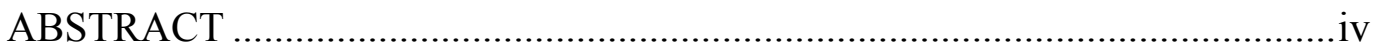

LIST OF FIGURES ................................................................................

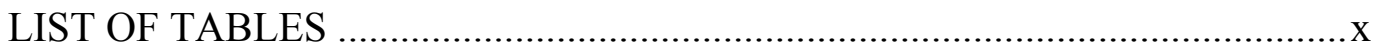

1. INTRODUCTION AND LITERATURE REVIEW ....................1

1.1 Tobacco Smoke Toxicity ..................................................2

1.2 Tobacco Smoke and Chronic Inflammatory Diseases..............3

1.3 Tobacco Smoke and Infectious Disease ...............................4

1.4 Periodontal Diseases......................................................6

1.5 Tobacco Smoke and Periodontal Disease.............................10

1.6 Tobacco Smoke and Periodontal Inflammation ....................12

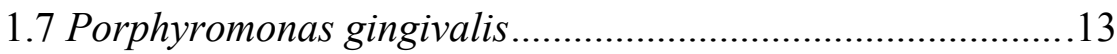

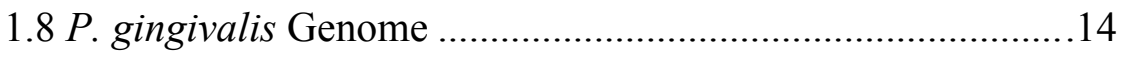

1.9 Clinical $P$. gingivalis Strain Variants ................................15

1.10 P. gingivalis and Periodontal Disease ...............................17

1.11 P. gingivalis Invasion, Proliferation, and Virulence ............18

1.11.1 P. gingivalis Invasion and Proliferation ...............18

1.11.2 P. gingivalis Capsule ........................................19

1.11.3 P. gingivalis Proteases (gingipains) ......................2 
1.11.4 P. gingivalis Fimbriae .21

1.11.5 P. gingivalis Lipopolysaccharide (LPS) ..............22

1.12 Tobacco Smoke and Bacterial Genomic Stability ...............23

1.13 Tobacco Smoke and P. gingivalis Genome, Physiology, and

Virulence ............................................................................24

1.13.1 P. gingivalis Genome and Physiology.................24

1.13.2 P. gingivalis Virulence ....................................26

1.14 Tobacco Smoke, P. gingivalis, and Periodontal Disease .....29

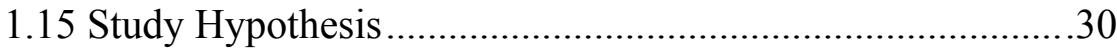

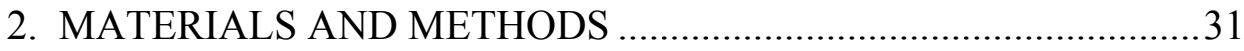

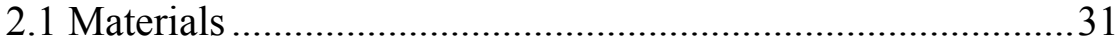

2.2 Bacterial Culture and In Vitro Modeling

of Tobacco Exposure ................................................................ 31

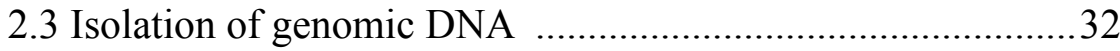

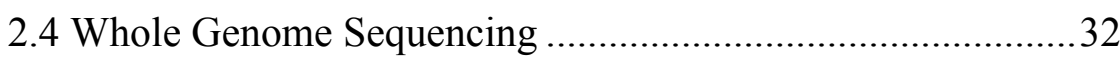

2.5 Confirmation of Mutations ..................................................... 33

2.5.1 Confirmation (i) - Visual Inspection......................33

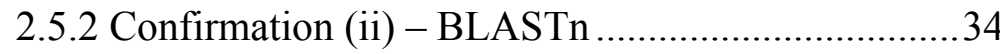

2.5.3 Confirmation (iii) - Allele Specific PCR ................34

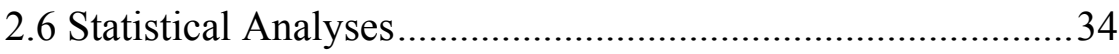

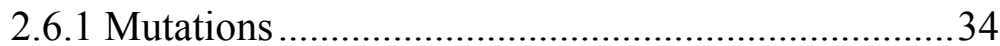

2.6.2 Allele Frequency/Read Variance............................35

3. CSE EXPOSURE PROMOTES GENETIC INSTABILITY AND BACTERIAL EVOLUTION. 
3.1 Results 36

3.1.1 CSE induces mutations in P. gingivalis ....................36

3.1.2 Confirmation of Mutations ..........................................37

3.1.3 CSE-exposure increases minor allele

Frequency (read variance) in P. gingivalis .........................39

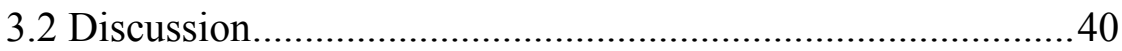

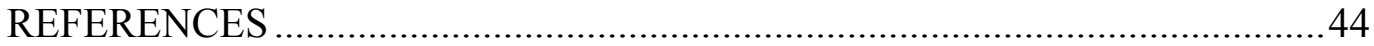

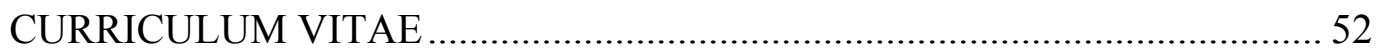




\section{LIST OF FIGURES}

1.4 Figure 1: Pathogenic colonization of the gingival sulcus and the host Immuno-inflammatory response.

3.2 Figure 2: 17 CSE-induced mutations

Throughout the $P$. gingivalis genome. 


\section{LIST OF TABLES}

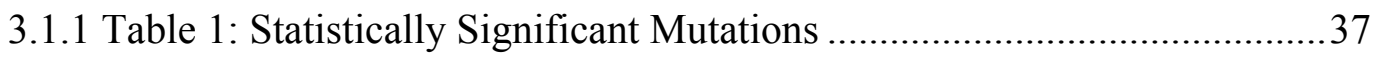

3.1.2 Table 2: Primer Design for Allele Specific PCR .........................................38

3.1.2 Table 3: Allele Specific Real-Time PCR $\Delta$ Ct Values .................................38

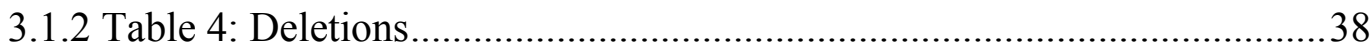

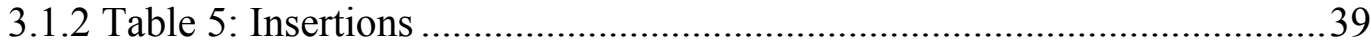

3.1.2 Table 6: Single Nucleotide Polymorphisms (SNPs) .................................39

3.1.3 Table 7: WGS Read Allele Variance........................................................40 


\section{CHAPTER ONE: INTRODUCTION AND LITERATURE REVIEW}

The use of smoking tobacco is one of the most major and preventable causes of premature mortality across the world $[1,30]$. Cigarette smoking and exposure to smoke is associated with chronic diseases, economic losses to society, and a substantial burden on the United States healthcare system, costing billions of dollars annually $[1,2]$.

From the years 2000-2004, it is estimated in the United States that cigarette smoking and cigarette smoke exposure resulted in 443,000 premature deaths, 5.1 million years of potential life lost, and $\$ 96.8$ billion in productivity losses annually [1]. One study estimated nearly 25 percent of all deaths in the age group 35-69 are directly attributed to smoking [30]. Studies have also implicated smoking as the primary causal factor for at least 30 percent of all cancer deaths as well as 80 percent of all deaths from chronic obstructive pulmonary disease and early-onset cardiovascular diseases [1].

Approximately one-third of the adult global population are tobacco users and 5-6 million people die annually due to tobacco-related illnesses [4]. While the use of smoking tobacco has declined in the United States in recent decades [30], global use has increased in developing nations [10]. Nearly 80 percent of annual tobacco related deaths occur in middle- to low-income countries [3]. 


\section{Tobacco Smoke Toxicity}

The serious ramifications of tobacco use come in the form of smoking related diseases. Tobacco smoke puts users at increased risk for a wide range of diseases such as; cardiovascular disease, oral, airway, and lung diseases, numerous cancers, immune disorders, persistent infections, and numerous chronic inflammatory diseases $[6,14,16$, $28]$.

Increased health risks are directly attributed to the high toxicity of tobacco smoke. The gaseous and particulate phases of cigarette smoke contain more than 4,500 chemicals which include 70 known carcinogens, as well as other reactive solids, oxidative species (free radicals), and mutagenic components [5-7]. Some of the more hazardous components of tobacco smoke include; ammonia, carbon monoxide, carbon dioxide, formaldehyde, acrolein, acetone, benzopyrenes, and hydroxyquinone as well as heavy metals including cadmium and arsenic $[5,6]$.

Smoking tobacco accounts for up to 85 percent of global tobacco production and use, and is the most hazardous form of tobacco use as observed by the more severe and diverse types of diseases when compared to smokeless tobacco [8].

Active smokers are not the only individuals at risk for adverse side effects from tobacco smoke. Cigarette smoke is a major environmental pollutant and exposure to second hand smoke has been linked to susceptibility to many of the same diseases associated with active smoking in both adults and children $[9,11,12,17]$

The causticity of tobacco smoke and its status as a ubiquitous environmental pollutant directly contribute to the global disease burden via its mutagenic capacity (explained in later section) as well as multiple adverse effects on the innate and adaptive 
immune systems, greatly increasing the risk of chronic infectious and inflammatory diseases $[5,13,15-22]$ as will be discussed in the following sections.

\section{Tobacco Smoke and Chronic Inflammatory Diseases}

Tobacco smoke puts users at an increased risk for developing chronic inflammatory diseases. While the mechanisms are not fully understood, studies have associated smoking with inflammatory conditions such as acute vascular diseases $[14,42]$, inflammatory bowel disorders [6,21] renal disease [19], pancreatitis [20] and periodontal diseases [41]. Studies also show smoke can deregulate endocrine and immune systems, predisposing users to autoimmune inflammatory disorders such as primary biliary disease (PBD), rheumatoid arthritis (RA), and multiple sclerosis (MS) $[22,43,44]$.

Evidence has shown tobacco smoke deregulates and increases the expression of several systemic pro-inflammatory markers indicated in chronic inflammation such as elevated levels of serum fibrinogen and plasma C-reactive proteins, as well as proinflammatory cytokines and chemokines, which in turn leads to increased levels of macrophages, monocytes, and neutrophils [44].

For example, smoke-induced stress on blood vessels increases vascular permeability and activates increased adhesion molecule expression, resulting in increased leukocyte adhesion and platelet aggregation eventually leading to atherosclerotic lesions and thrombus formations greatly increasing the risk of heart attack and/or stroke $[14,42,48,49]$. 
Pulmonary dysfunction and chronic inflammation of the airways such as emphysema and chronic obstructive pulmonary disorder (COPD) are also elicited by tobacco smoke [47]. Smoke exposure on airway epithelia increases pro-inflammatory cytokine expression, induces rapid influx of activated neutrophils and macrophages and formation of free radicals, as well as release of degradative enzymes such as neutrophilic elastase and matrix metalloproteases (MMPs) [40].

\section{Tobacco Smoke and Infectious Diseases}

Smokers as well as those exposed to environmental (second-hand) smoke are more susceptible to contracting infectious diseases than non-smokers, such as respiratory tract infections, pneumonia, tuberculosis, meningitis, bacterial sexually transmitted diseases, and periodontal diseases to name only a few $[17,25]$. While there is a robust amount of evidence correlating the effects of smoking adversely affecting human health, there is a gap in knowledge, mechanistically to explain such phenomena [102].

It has been hypothesized that smoking increases the risk of infection by three general mechanisms: tobacco-induced host physiological and structural changes, deregulation of innate and adaptive immune systems, and increasing bacterial virulence [17]. There is much evidence to support this hypothesis.

Cigarette smoke components and their metabolites such as acrolein, acetaldehyde, formaldehyde, and free radicals are implicated in contributing to structural changes observed in the respiratory tract, aiding bacterial colonization and infection $[100,101]$. Such changes include peribronchiolar inflammation and fibrosis, increased mucosal 
permeability and impairment of the mucociliary clearance. These structural changes decrease the ability of the host to clear pathogens from the airways, aiding bacterial colonization and infection [103].

Various structural changes can also be induced by cigarette smoke [17]. Clinical evidence has shown cigarette smoke exposure causes cerebral vessels to dilate [95], while causing peripheral arterial vessels constrict [46]. In the periodontal tissues, cigarette smoke has been shown to suppress angiogenesis [57].

Cigarette smoke is also highly implicated in deregulating the innate immune system. Studies have shown tobacco smoke to decrease phagocytic activity and generation of reactive-oxygen species in neutrophils and monocytes, suppressing their responsiveness and bacterial-killing capacity, and consequently aiding pathogenic colonization and infection [104-106]. Tobacco smoke also reduced dendritic cell antigen-presenting and co-stimulatory molecules, and reduced capacity for antigen uptake and production of T-cell stimulatory cytokines in murine models [107].

Adaptive immunity is also compromised upon exposure to tobacco smoke. Reduced serum levels of Immunoglobulin G ( $\operatorname{IgG})$, an important anti-microbial antibody, have been detected in smokers when compared to non-smokers [96,97]. T-cell proliferative responses have also shown to be suppressed in smokers. Studies have shown a decrease in the ratio of CD4 to CD8 lymphocytes in heavy smokers. This is explained by a decrease in CD4 lymphocytes while there is a simultaneous increase in CD8 lymphocytes [98,99]. Because CD4 lymphocytes stimulate B-cell proliferation and differentiation as well as immunoglobulin synthesis, the decrease of CD4 lymphocytes in 
heavy smokers may be a possible explanation for the observed reduced serum IgG levels in smokers [25].

Smoke may also induce increased bacterial virulence, contributing to increased susceptibility of infection [17]. For example, smoke exposure induced increased adhesion of respiratory pathogens to airway epithelial cells in smokers [108]. Smoke can also induce shifts in microbial populations allowing pathogens to proliferate [109]. While there is still much research that needs to be conducted on the subject, there is convincing evidence that tobacco smoke can both genotypically and/or phenotypically alter pathogens and increase virulence. The effects of tobacco-induced alterations on the genome, physiology, and virulence of the periodontal pathogen Porphyromonas gingivalis will be discussed in detail in later sections.

\section{Periodontal Diseases}

Periodontal diseases are both infectious and inflammatory. Data from the World Health Organization suggests that 5-20 percent of the adult global population suffers from severe periodontitis and that most children and adolescents will exhibit signs of gingivitis at some point in their lifetime [130]. A study using data from the 2009 and 2010 National Health and Nutrition Examination Survey (NHANES) estimated the prevalence, severity, and extent of periodontitis in the adult U.S. population. The results indicated that roughly 47 percent adults aged 30 years or older, had periodontitis, distributed as 8.7 percent, 30 percent, and 8.5 percent with mild, moderate, and severe periodontitis, respectively. For adults aged 65 and older, 64 percent 
had either moderate or severe periodontitis. This survey provides direct evidence of the high burden of periodontitis in the United States [34].

Bacteria in the oral cavity often proliferate in an attached multispecies biofilm community, commonly known as dental plaque. The composition of the microbiota in biofilms can be altered due to factors such as diet, host genotype, antibiotic ingestion, pathogen infection, and other environmental effects, which can greatly affect host physiology, metabolism, and immune systems [117]. Periodontal disease and the resulting inflammation is caused when Gram negative oral pathogens such as $P$. gingivalis, Treponema denticola, and Tannerella forsynthia invade the gingival sulcus between the junctional epithelium below the gingival margin and the clinical root of the tooth, altering the commensal biofilm composition, and challenging the host immune system $[24,50]$ as shown in Figure 1. Subgingival colonization by these periodontal pathogens is aided by the expression of several immune subverting virulence factors, which will be discussed in a later section. 


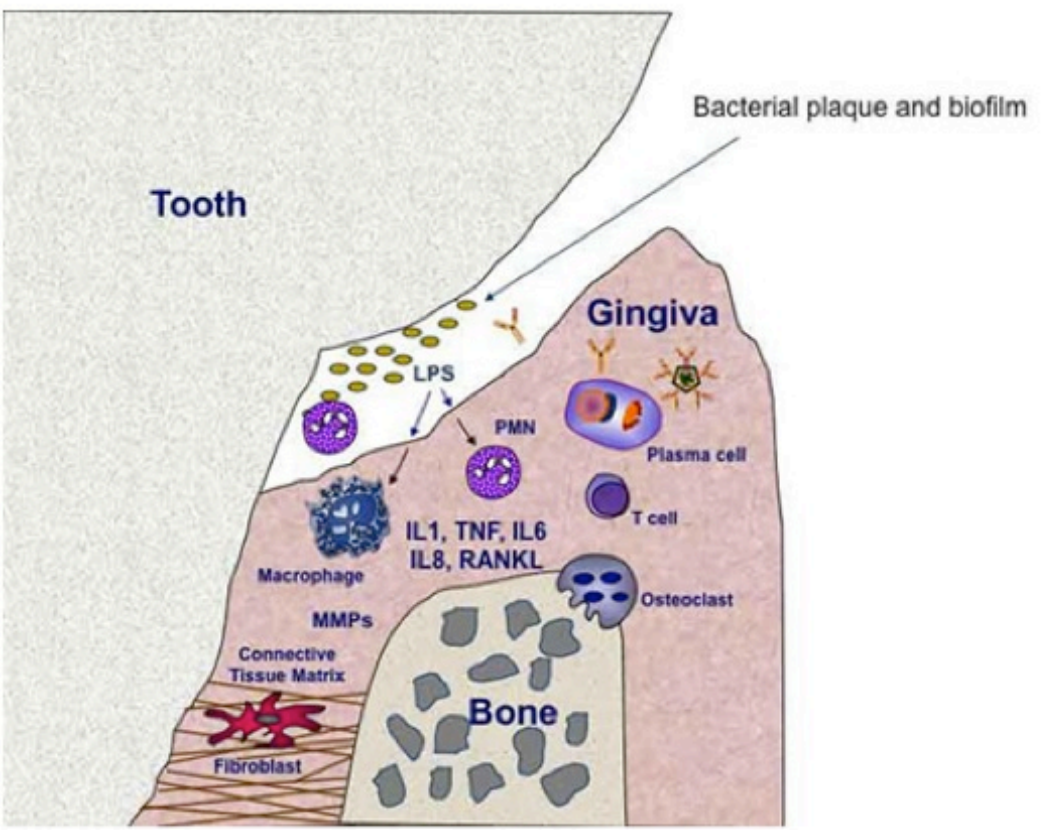

Figure 1: Pathogenic colonization of the gingival sulcus and the host immunoinflammatory response.

Photo courtesy of: www.hopkinsarthritis.org

Figure 1 shows what occurs during periodontal disease - accumulation of pathogenic bacteria and biofilm formation that develops in the subgingival pocket. The release of multiple pro-inflammatory cytokines (IL-1, TNF, IL-6, etc) are detectable and are expressed in the gingival tissue as well as released into the gingival crevicular fluid activating neutrophils, macrophages, and recruiting them to the site of infection eventually leading to connective tissue matrix degradation and osteoclast activation which leads to the degradation of bone.

There are progressive stages of periodontal disease that are characterized by the severity of hard and soft periodontal tissue damage, such as bleeding on probing, pocket depth, and gingival and alveolar bone resorption. Gingivitis is the initial stage of periodontal disease and is characterized by minor redness, swelling, and bleeding on 
probing. The minor inflammation of gingivitis is reversible and does not cause any lasting or permanent damage [27]. However, if left untreated, gingivitis can progress into the more severe form of periodontal disease, periodontitis. Periodontitis is characterized by irreversible inflammatory damage of the periodontal tissues such as detachment of the periodontal ligament, gingival resorption, and alveolar bone loss, eroding the supporting structures of the teeth resulting in tooth mortality and tooth loss over time $[23,24]$.

The severity and progression of the inflammatory immune response varies depending on host-specific factors. Things such as age, race, and immune abnormalities are just a few of the important factors that affect the host immuno-inflammatory response [29].

The colonization of the sub-gingival pocket by Gram-negative periodontal pathogens stimulates secretion of pro-inflammatory cytokines such as IL-1, IL-6, IL-8, TNF and RANKL from host oral epithelial cells [51] as shown in Figure 1. Proinflammatory cytokine secretion causes immediate swelling and redness of the periodontal tissues. Inflammation becomes more severe as pathogens proliferate promoting further, possible permanent tissue damage [23, 24, 27].

Bacterial persistence and prolonged cytokine secretion activate osteoclasts in the host alveolar bone [37]. When osteoclasts become activated, bone matrix is broken down causing irreversible bone resorption from the alveolar crest. If left untreated, bone loss will continue apically toward of the root of the tooth [31]. The destruction of the supporting tooth structure consequently causes loss of attachment resulting in tooth mobility and eventual loss of the tooth $[23,24]$. 


\section{Tobacco Smoke and Periodontal Disease}

Tobacco smoke is a major environmental pollutant and significant risk factor for periodontitis. Accordingly, smokers have a high predisposition of developing plaqueinduced periodontal diseases and consequently a higher prevalence $[29,32,33]$. Tobacco smoke is a major risk factor for periodontal disease because the oral cavity is the entryway for smoke into the airways and therefore naturally has the highest concentration of smoke components, especially in the gingival crevicular fluid (GCF) excreted from the gingival sulcus [93].

Evidence suggest high concentrations of tobacco smoke components induces a shift in periodontal plaque composition from one that is mainly constituted of Gram positive, aerobic, commensal bacteria to one that contains more Gram negative, anaerobic pathogens, such as $P$. gingivalis [50].

Studies show a clear dose-dependent relationship between smoking and periodontal disease [35]. High levels of tobacco smoke inhalation are associated with faster disease progression and more severe periodontal damage. Clinical evidence shows increased gingival recession and alveolar bone loss, greater periodontal ligament (PDL) attachment loss and deeper gingival pocket formation that is responsible for increased tooth mobility and tooth loss at an earlier age than non-smokers [36,37]. It has also been shown that patients who suffer from periodontal disease are much more likely to be refractory to treatment if they are active smokers, when compared against non-smokers [36]. 
Various studies have been conducted which give insight into the link between tobacco smoke and periodontal disease. Nicotine, a major smoke component has been shown to induce periodontal collagen degradation by increasing expression of the collagenase matrix metalloproteases (MMPs), which may result in detachment of the periodontal ligaments [54]. Several studies have also indicated nicotine and one of its major metabolites cotinine, adversely affect human periodontal fibroblasts (PDFs) decreasing their levels of attachment, proliferation, and chemotactic responsiveness in a dose-dependent manner $[39,52]$. One study shows, upon exposure to nicotine stress periodontal fibroblasts increase c-fos expression, to protect against cellular damage [55]. This same study suggests that the nicotine-dependent stress-specific expression of the cfos gene correlates with cellular thiol levels in human PDFs. Thiols are exceedingly important in that they act as an antioxidant defense system to protect the cell against oxidative damage.

Acrolein is another pernicious smoke component and major player in the destruction of periodontal tissues. In a study conducted by Chang et al. [55], it was shown that acrolein significantly depleted intracellular thiol levels in PDFs. Therefore, decreased levels of thiol make the PDFs much more susceptible to damage by oxidants contained in tobacco smoke. The same study also showed that acrolein can suppress PDF proliferation by as much as fifty percent, resulting in significant attachment loss of the periodontal ligaments. 


\section{Tobacco Smoke and Periodontal Inflammation}

Despite being more susceptible to periodontitis and exhibiting faster disease progression and severity, chronic smokers lack the clinically overt inflammatory response to bacterial plaque that non-smokers exhibit such as redness, swelling, bleeding on probing and reduced pro-inflammatory indicators [56]. As discussed in previous sections, tobacco smoke is a major environmental toxin and as such, has the ability to alter the user's inflammatory responses by deregulating the innate and adaptive immune systems.

Evidence suggests that tobacco smoke deregulates important sequential adhesion molecule cascades that are critically important for leukocyte recruitment and causes inappropriate leukocyte activation [57]. Therefore, normal inflammatory reactions may be compromised. Cigarette smoke also induces local suppression of angiogenesis, providing a possible explanation for reduced amounts of bleeding $[56,58]$.

All of these observations make it apparent that tobacco smoke plays an important role in the pathogenesis and severity of periodontal disease. However, the mechanisms by which tobacco smoke masks periodontal inflammation warrants further investigation [56]. Much of the research on the matter has been directed towards the host response to smoking and periodontal infection. However, it is poorly understood how tobacco smoke affects periodontal pathogens and what affect this has on their virulence in pathogens such as $P$. gingivalis. 


\section{Porphyromonas gingivalis}

Porphyromonas gingivalis is an opportunistic, Gram-negative, assacharolytic, anaerobic oral pathogen highly implicated in the pathogenesis of periodontitis and systemic sequelae. Socransky et al. 1998 characterized periodontal microbial communities based on cluster analysis, community ordination and associated disease severity. The periodontal bacteria were then categorized into five separate color-based complexes which increase in severity of disease association in the following order; purple, yellow, green, orange, and red. P. gingivalis is a member of the "red complex" which also includes $T$. denticola and $T$. forsythia.

Numerous studies have detected these three oral pathogens as being strongly associated with each other and with severe disease sites [66]. Due to the ease of growth and ability to be genetically manipulated, $P$. gingivalis has become the most well studied out of the three oral bacteria in the red complex [74].

Further analysis of $P$. gingivalis has led to its designation as a keystone pathogen. A validated murine model study [112] provides evidence that $P$. gingivalis, even at low abundance has the ability to significantly induce alterations to host commensal microbiota in a way supports its own viability while promoting disease pathogenesis. In these models, specific pathogen free (SPF) mice inoculated with $P$. gingivalis caused significant bone loss which is a defining characteristic of periodontal disease. In contrast, germ free (GF) mice inoculated with a $P$. gingivalis mono-infection saw no significant bone loss indicating that $P$. gingivalis alone cannot induce periodontitis. This study indicates that rather than directly attacking host periodontal tissues, $P$. gingivalis 
contributes to disease indirectly by significantly altering the total commensal microbial load and biofilm composition, which overwhelms normal host tissue-protective mechanisms and homeostasis, resulting in an immune-inflammatory response, promoting disease pathogenesis.

\section{$P$. gingivalis Genome}

Nelson et al. [110], sequenced the complete 2,343,479-bp genome of $P$. gingivalis strain W83, a highly virulent strain used in experiments conducted for this thesis. The W83 genome contains 2,015 genes, encoding 1,990 and has an average G/C content of 48.3\%. There are four ribosomal operons and two structural RNA genes, as well as 53 tRNA genes with specificity for all 20 amino acids. Analysis revealed a total of 1,990 open reading frames (ORFs) approximately $85 \%$ of the genome. Of these ORFs, 1,075 were assigned biological roles, 184 were conserved hypothetical proteins or conserved domain proteins, 208 had unknown functions, and 523 encoded hypothetical proteins.

Repetitive elements were revealed including DNA repeats \{direct repeats and clustered regularly interspaced short palindromic repeats (CRISPRs)\} as well as transposable elements \{nsertion sequences (ISPg 1-11) and miniature inverted-repeat transposable elements (MITE239, MITE464, MITE700)\}. Repetitive and transposable elements occupy approximately $6 \%$ of the genome. It was revealed that 96 complete or partial copies of IS elements are present within the genome. However, it was indicated they are rarely found in functional genes and instead are inserted almost exclusively into intergenetic regions (IGRs) and other copies of transposable elements. 
Further analysis indicated 21 areas of the genome that displayed atypical nucleotide composition based on increased and/or decreased levels of G/C content when compared to the rest of the genome. The investigators believed a variety of genes could possibly have been acquired through lateral gene transfer and are encoded in these regions. These genes include restriction system proteins, hemagglutinin proteins $\mathrm{B}$ and $\mathrm{C}$, numerous capsular biosynthesis proteins, 20 transposase genes, and two large mobile elements.

\section{Clinical $P$. gingivalis Strain Variants}

Genotypic characterization of $P$. gingivalis strains has revealed extensive genetic variability [72]. A study by Griffen et al. [114], used heteroduplex analysis of the ribosomal internal spacer region (ISR) between $16 \mathrm{~S}$ and $23 \mathrm{~S}$ ribosomal genes of $P$. gingivalis to detect strain variants as well as determine if all strains exhibit similar strengths of association with periodontal disease or rather if there are more- and lessvirulent strains. P. gingivalis samples were collected from patients with clear indicators of periodontal disease as well as age-matched, healthy, control patients. Analysis revealed eleven $P$. gingivalis heteroduplex types, of which six types had sufficient numbers for statistical analysis. It was determined type hW83 was the most strongly associated with periodontal disease. Additionally, types h49417 and hHG1691 were also significantly associated with periodontitis but with lower odds ratios than hW83. The remaining three types $\mathrm{h} 23 \mathrm{~A} 4, \mathrm{~h} 381$, and hA7A1 were not significantly associated with 
disease pathogenesis. The results of these analyses indicate there are in fact differences in virulence between clinical strains of $P$. gingivalis.

A more recent study conducted by Igboin et al. [113] confirmed the previous study's findings as well as used ISR heteroduplex analysis to determine worldwide genetic variability and distribution of $P$. gingivalis. In this study, samples were taken from patients at 3 U.S locations (Columbus, Ohio/San Antonio, Texas/San Francisco, California) as well as from foreign college students entering the U.S. who had arrived no more than 30 days prior to taking plaque samples. Heteroduplex analysis confirmed the six predominant heteroduplex types \{W83 (including strain W50), 23A4, A7A1, 381 (including strain ATCC 33277), HG1691, and ATCC49417\} of the previous study in all U.S. locations as well as foreign continents, with 14 rare heteroduplex types showing up in only one or a few locations.

Furthermore, investigators used microarray-based comparative genomic hybridization $(\mathrm{CGH})$ to analyze whole genome variability of the major heteroduplex strains. Using strain W83 as the control, it was found that the control genome was highly conserved among the major heteroduplex types ranging between $0 \%$ variability when compared with strain W50 to 5.1\% when compared to strain ATCC 33277 . Both ISR and CGH revealed strains ATCC 49417 and W50 to be very closely related to strain W83, respectively. 


\section{$P$. gingivalis and Periodontal Disease}

Studies have shown more than 700 species of bacteria can colonize the oral cavity, while only a small number are associated with disease [65]. Of these, $P$. gingivalis is strongly implicated in the development and progression of periodontal disease. It is widely believed that polymicrobial interactions within dental plaques are may be responsible for the initiation and progression of inflammatory periodontal diseases [119]. Unfavorable alterations in periodontal biofilm communities, such as pathogenic outgrowth of a keystone species like $P$. gingivalis, deregulate host homeostasis [112,121-122]. P. gingivalis exhibits aggregative properties and several virulence factors which aid in colonization and tissue destruction such as fimbriae, proteases, lipopolysaccharides, and capsular polysaccharides [119]. Based on these properties and clinical observations, $P$. gingivalis is considered an opportunistic pathogen that is significantly associated with periodontal disease [65]. It is often isolated from plaque biofilms removed from tooth surfaces in individuals with periodontal disease (79$90 \%)$ while rarely found in healthy individuals (10-25\%) [113].

Several studies have linked the association of $P$. gingivalis with periodontal disease. P. gingivalis has been positively correlated with gingival pocket depth formation in humans indicating there is an approximate 10-fold increase of $P$. gingivalis cells per 1millimeter of increased pocket depth in diseased sites [116]. A review of several animal studies revealed $P$. gingivalis induced inflammatory immune responses and periodontal bone loss in these models [120]. It has also been shown that reducing the numbers of $P$. gingivalis cells via clinical scaling and root planing reduced clinical symptoms of 
periodontal disease such as gingival redness, bleeding on probing, and pocket depth [123].

\section{P. gingivalis Invasion, Proliferation, and Virulence}

\section{P. gingivalis Invasion and Proliferation}

$P$. gingivalis has the ability to invade host periodontal tissues while evading host defenses. In doing so, it causes deregulation of the host immuno-inflammatory responses [50]. The following sections will discuss the invasiveness of $P$. gingivalis and its ability to evade host defenses as well as the role of its major virulence factors including the capsule, gingipains, fimbriae, and lipopolysaccharides (LPS).

$P$. gingivalis is able to actively invade host cells such as human gingival epithelial cells, where it can avoid immune surveillance, replicate, and stay viable within the host cell $[62,67]$. $P$. gingivalis also has the ability to invade macrophages, but does not replicate as frequently as within other cells [64]. P. gingivalis uses its major fimbriae to invade host cells. These major fimbriae bind with host cell-surface $\beta 1$ integrins where $P$. gingivalis can then rearrange the host cell's cytoskeleton allowing it to internalize and move from cell to cell via cytoskeleton bridges [63]. Evidence has shown that $P$. gingivalis remains viable within host cells as there is no evidence of necrosis or apoptosis. P. gingivalis has the ability inhibit gingival epithelial cell apoptosis induced by ATP ligation of $\mathrm{P}_{2} \mathrm{X}_{7}$ receptors. This is accomplished through secretion of an ATPhydrolyzing enzyme, suppressing host cell ATP-dependent apoptosis [62]. The ability of 
P. gingivalis to internalize itself in host cells and remain viable while subverting immune surveillance greatly influences its survival and proliferation in the periodontal pocket.

\section{P. gingivalis Capsule}

Strains of $P$. gingivalis are very heterogeneous and some of these strains, including W83, produce an extracellular capsular polysaccharide (CPS), also known as its K-antigen or serotype. There are at least six different known serotypes, which are characterized by the capsules ability to generate systemic $\operatorname{IgG}$ antibody responses.

Encapsulated strains of $P$. gingivalis are shown to be highly invasive, while being able to evade the host pro-inflammatory immune response [50]. In murine models, encapsulated strains of $P$. gingivalis were associated with increased abscess formation and spreading infection when compared to non-encapsulated strains, which only induced localized abscesses [68].

In a study by Brunner et al. [61], the role of the $P$. gingivalis W83 capsule in evading the innate immune response was studied. An $\operatorname{esp} C$ gene-knockout mutant was constructed for $P$. gingivalis W83; espC is located at the end of the capsular synthesis locus inhibiting formation of the capsule. The wild-type strain and the espC mutant challenged human gingival fibroblasts (HGFs) and the expressions of IL-1 $\beta$, IL-6, and IL-8 transcription levels were measured. Results indicated high cytokine and chemokine transcription levels were found when the HGFs were challenged with the $\operatorname{esp} C$ knockout than with the wild-type strain. This evidence suggests that the capsule may help mask $P$.

gingivalis from the host pro-inflammatory immune response, helping to promote virulence. 
Such a mechanism is especially important in the case of infectious chronic inflammatory diseases such as periodontal disease in which evading the host immune response helps $P$. gingivalis proliferate and persist while avoiding immune clearance [59]. However, the potential involvement of the capsule in the overall deregulation of the host response needs further study [50].

P. gingivalis Proteases (gingipains)

P. gingivalis produces trypsin-like cell surface cysteine proteinases, also known as gingipains. Gingipains can account for up to $85 \%$ of the proteolytic activity of $P$. gingivalis. Based on substrate specificity, they are divided into arginine-specific (Arg-X) and lysine-specific (Lys-X) gingipains coded by the $\operatorname{rgp} A, \operatorname{rgp} B$, and $\operatorname{kgp}$ genes, respectively [69].

Gingipains play an important role in the virulence of $P$. gingivalis while also deregulating the host immuno-inflammatory response. Gingipains are capable of cleaving T-cell receptors such as CD4 and CD8, thereby greatly inhibiting cell-mediated immunity [71]. They also have the ability to degrade cytokines and complement pathway proteins conceding increased resistance to bacterial clearance from the host immune response [50].

Aside from evading immune surveillance, gingipains may be directly involved in the ability of $P$. gingivalis to invade host cells. Evidence has shown $r g p$ to mediate $P$. gingivalis adherence to oral epithelial cells. Evidence also shows once $P$. gingivalis has invaded the host cell, expression of gingipains is reduced [70]. 
Gingipains may also affect vascular permeability and the blood coagulation system causing increased bleeding at the periodontal site perpetuating inflammation and gingival edema [50].

\section{$\underline{P}$ gingivalis Fimbriae}

The major fimbriae of $P$. gingivalis are thin, hair-like filamentous cell-surface structures encoded by the fimA BCDE operon. P. gingivalis fimbriae facilitate its ability to adhere to salivary proteins, extracellular matrix, oral epithelial cells, and bacteria of the same or other species. Through its fimbriae, $P$. gingivalis can adhere itself to early colonizing bacteria, and participate in the developing biofilm structure making it essential for invading and colonizing the host [74].

There are both major and minor fimbriae. Studies show major fimbriae play an important role in colonization and invasion, while minor fimbriae are indicated in activating the host inflammatory response [50]. Based on genomic variations of the fim $A$ gene, major fimbriae have been classified into six types (Type I-V, Ib) [73,74]. Minor fimbriae are encoded from the $m f a$ gene ( $m f a 1, m f a 2)$ [78]. Type II, major fimbriae is found to be the most virulent and commonly associated with severe periodontal disease $[75]$.

Alveolar bone resorption is a hallmark of periodontitis. Murine models of periodontitis directly indicate $P$. gingivalis major (type II) and minor ( $m f a l$ ) fimbriae in the induction of alveolar bone destruction [77]. Other characteristics of major and minor fimbriae are the capacity to evoke a pro-inflammatory cytokine response (IL-1, IL-6, IL8 , and TNF- $\alpha$ ) from various host cells as well as expression of matrix metalloproteases 
[50]. Major fimbriae induce increased expression of adhesion molecules from host oral epithelial cells promoting further adhesion and invasion of host cells by $P$. gingivalis [79]. P. gingivalis fimbriae have also proven to be an important virulence factor for survival, interacting with complement receptor 3 (CR3) inhibiting IL-12 secretion from host immune cells increasing resistance against immune clearance [76].

P. gingivalis Lipopolysaccharide (LPS)

Toll-like receptors (TLRs) play a pivotal role in initiating host immune responses and are implicated to be involved in the development of many infectious diseases, such as periodontitis [80]. P. gingivalis LPS is a key inflammatory mediator. Studies have shown $P$. gingivalis LPS to be recognized by TLR2 and TLR9, while there is debate over TLR4 recognition $[80,81]$. P. gingivalis LPS stimulates pro-inflammatory cytokine secretion from host immune and oral epithelial cells, including IL-1, IL-6, IL-8, IL-18, and TNF- $\alpha$ [50]. It is also implicated in alveolar bone loss [82], as well as increasing risk the risk of systemic disease such as atherosclerosis [83].

P. gingivalis LPS is a weaker inducer of inflammation and pro-inflammatory cytokine secretion than LPS of other Gram-negative bacteria, such as Escherichia coli [84]. While most gram-negative LPS's are strong TLR4 inducers, $P$. gingivalis LPS primarily activates TLR2 and may even serve as an antagonist to TLR4 $[85,86]$. Evidence indicates this is due to structural LPS heterogeneity found within the acylation patterns of the lipid A portion of $P$. gingivalis LPS. There are two known forms of $P$. gingivalis LPS lipid A - penta-acylated and tetra-acylated. Studies show penta-acylated lipid A acts as an agonist on TLR4, while tetra-acylated lipid A acts antagonistically 
against TLR4, modulating the host immune response [85, 87]. Variations in acyl chain length have also shown to alter host immuno-inflammatory activity [88]. These alterations to the lipid A portion of $P$. gingivalis LPS, as well as the host immune response can be strain specific or environmentally induced by things such as hemin concentration $[88,89]$.

\section{Tobacco Smoke and Bacterial Genomic Stability}

A study conducted by Aufderheide et al. [90] examined the bacterial mutagenic and cytotoxic capabilities of cigarette mainstream whole smoke and its gas/vapor phase using a modified Ames assay (bacterial reverse mutation assay). Standard $S$. typhimurium bacterial tester strains were used: TA98, TA100, TA1535, TA1537, TA1538, TA102, as well as descendant strains of TA98 (YG1021, YG1024, YG1041) and TA00 (YG1026, YG1029, YG1042). WP2uvrApKM101, an E. coli strain, was also used.

The mutagenicity of WS smoke was detected using (TA1538) TA98, (TA1535) TA100 strains and their YG descendants as well as WP2uvrApKM101 in the presence of S9 mix (metabolic activating system) and the plasmid pKM101, requiring a frame-shift mutation. To detect mutagenicity in GVP, TA100 and descendent strains as well as WP2uvrApKM101 were used in the presence of the pKM101 plasmid and the absence of S9 mix, requiring a missense mutation. Based on the addition of S9 mix to WS analysis, investigators implicated that many of the components need to be metabolized before 
exerting mutagenic activity. Oppositely, the absence of S9 in the GVP analysis indicates many of the mutagenic components are direct acting.

The results of WS analysis revealed that all strains showed genetic mutations (induction of revertants). Mutagenic activity increased in a dose-dependent manner to a maximum point that varied among different strains after which point the WS induced toxic effects. GVP analysis also revealed genetic mutations. Missense mutations were detected in WP2uvrApKM101 as well as strains TA1538, TA100 and its YG descendants apart from YG1026.

Further analysis using several brands of cigarettes revealed that increased levels of tar increased the mutagenic capacity of the cigarette smoke in most cases. Investigators determined that tar content alone is not solely responsible for increased mutation. Rather, the composition of the tar such as different types of tobacco, nitrate levels, etc. as well as cigarette components and function such as filters and ventilation are responsible for mutagenic capacity [90]. These findings are in line with previous studies $[91,92]$ conducted to determine the mutagenic capabilities of cigarette smoke.

The effects of tobacco smoke on the genomic stability and virulence of the periodontal pathogen $P$. gingivalis is explained in the following sections.

\section{Tobacco Smoke and $P$. gingivalis Genome, Physiology, and Virulence}

\section{P. gingivalis Genome and Physiology}

The previous sections have discussed the toxicity of tobacco smoke and the impact tobacco smoke has on deregulating host immuno-inflammatory responses to 
bacterial infections such as periodontal disease. However, little is known about the effects tobacco smoke has on bacterial physiology and genomic stability and the role it plays in disease pathogenesis, such as with the periodontal pathogen $P$. gingivalis.

A study by Bagaitkar et al. [115] investigated the effects of cigarette smoke extract (CSE) on gene expression in $P$. gingivalis. Using microarray analysis, it was discovered that $6.8 \%$ of the $P$. gingivalis genome was differentially expressed upon exposure to CSE. A total of 104 genes were significantly differentially expressed by a minimum of two-fold or greater. Of which, CSE induced expression of 58 genes while suppressing 46 .

Analysis revealed many of the genes induced by CSE had known or putative functions, several of which were located within operons or were functionally related. Of the 58 genes induced by CSE, two genes were identified in the major fimbrial operon, as well as an operon encoding outer-membrane antigenic lipoproteins. Groups of functionally related genes were also identified such as in DNA replication and repair genes ( $\mathrm{ruvC}, \mathrm{radC}$, and a putative integrase), genetic insertions and transpositions (traE, $\operatorname{traF}$, and $\operatorname{traK}$ ), as well as insertion sequences (ISPg3, ISPg5, and ISPg6). Several other transfer $(\operatorname{tra})$ genes were up-regulated but fell below the two-fold threshold. Numerous genes involved in host-pathogen interactions and virulence were also up-regulated such as proteases (tpr, trpT, and PG0317), two putative lipoproteins required for major fimbriae assembly, and the major 61 and $63 \mathrm{kDa}$ surface antigens as well as other cell surface polypeptides.

Of the 46 genes suppressed by CSE exposure, many encoded hypothetical proteins whose functions are unknown. However, two genes were located within the 
capsular synthesis locus; capK, PG0111 (polysaccharide synthesis gene), and PG0108 (polysaccharide transport protein). Additionally, two other capsular synthesis genes were also down-regulated upon CSE exposure but fell just below the two-fold limit. $h m u R$, a tonB-dependent hemoglobin receptor was also down-regulated as well as fimS, a sensor histidine kinase which is believed to be involved in regulating the expression of the major and minor fimbrial operons.

In addition to microarray analysis, a cellular membrane fragment was analyzed by SDS gel electrophoresis, which after analysis revealed overexpression of three bands that were identified as RagA, RagB, and a putative lipoprotein (PG1079) that is believed to be co-expressed with the minor fimbrial antigen.

It was concluded that the a number of the genes differently regulated by CSE exposure are associated with aspects of $P$. gingivalis virulence such as fimbrial and capsular operons, transcriptional regulators, transport proteins, proteases and cell capsule proteins as well as up-regulation of several insertion sequence (IS) elements induced by CSE promote genetic rearrangements. It is believed that the genomic response to CSE exposure may partially explain the reduced inflammatory potential and altered hostpathogen interaction of $P$. gingivalis exhibited in smokers with periodontal disease.

\section{P. gingivalis Virulence}

The previous section described how $P$. gingivalis adapts to tobacco-induced stress by altering the expression of several genes and outer membrane proteins involved in virulence, which may serve to lower its inflammatory potential while increasing its virulence. The microarray study revealed that CSE induced proteolytic activity, which 
may contribute to increased virulence. Also, major fimbrial genes (PG2133, PG2134) were induced, while several genes associated with capsular biosynthesis (capK, wecC, PG0117, PG0118) were suppressed [115,126].

P. gingivalis colonization of the oral cavity is dependent upon its interaction with other plaque bacteria, including Streptococcus gordonii [119,124]. Another study by Bagaitkar et al., 2011 hypothesized that cigarette smoke promoted $P$. gingivalis-S. gordonii biofilm formation in a FimA-dependent manner. The previous microarray analysis revealed while major fimbriae expression was significantly increased, minor fimbriae function was unaffected [115]. Experimental evidence revealed that $P$. gingivalis biofilms grown in the presence of CSE exhibited a significantly lower proinflammatory capacity (TNF, IL-6) and bound more strongly to immobilized rGAPDH, the cognate FimA ligand on $S$. gordonii, than control biofilms in a dose-dependent manner. Nevertheless, a peptide representing the minor fimbrial binding site on $S$. gordonii, (SspB), completely inhibited dual species biofilm formation. Investigators concluded that CSE likely augments $P$. gingivalis biofilm formation by increasing major fimbrial avidity, which, in turn, supports initial interspecies interactions and promotes subsequent high affinity Mfa1-SspB interactions driving biofilm growth. CSE induction of $P$. gingivalis biofilms of limited pro-inflammatory potential may explain the increased persistence of this pathogen in smokers [124]. Additionally, evidence suggests $P$. gingivalis capsule production decreases ability of biofilm growth [125]. P. gingivalis capsular biosynthesis genes (capK, wecC, PG0117, PG0118) were suppressed [115,126], which may also serve to help promote $P$. gingivalis biofilm growth and proliferation. 
Lipid A heterogeneity of $P$. gingivalis lipopolysaccharides (LPS) are also implicated in $P$. gingivalis virulence and inflammatory potential [128]. Evidence suggests tobacco smoke has the ability to induce alteration in the Lipid A region of $P$. gingivalis LPS reducing inflammatory potential, therefore increasing virulence [127].

A study by Buduneli et al. [127] analyzed fatty acid profiles of periodontal bacteria from salivary samples from individuals with and without periodontal disease, with a subset of active smokers and non-smokers in each group. It was revealed that the acyl chain length of 3-OH fatty acids attached to the lipid A portion of bacterial LPS significantly affects inflammatory response in periodontal bacteria. Short straightchained fatty acids (12-14 carbons in length) had the highest potency in instigating an inflammatory response, while longer chains (17 carbons in length) showed significantly reduced inflammatory potential.

A study by Herath et al. [129] also gives evidence that structural lipid A heterogeneity in $P$. gingivalis can reduce its inflammatory potential by examining how $P$. gingivalis LPS lipid A heterogeneity differentially modulates the expression of IL-6 and IL-8 in human gingival fibroblasts (HGFs). HGFs were stimulated with P. gingivalis tetra-acylated LPS (1435/1449) and penta-acylated LPS (1690) in a dose dependent manner. Results indicated that penta-acylated LPS (1690) significantly upregulated the mRNA and protein expression of IL- 6 and IL-8, whereas the tetra-acylated LPS (1435/1449) did not induce significant host response. Because tobacco smoke has the ability to alter acyl chain length [127], it can be easily hypothesized that tobacco smoke may also influence $P$. gingivalis LPS lipid A acylation. P. gingivalis LPS lipid A heterogeneity may differentially modulate pro-inflammatory cytokine expression, 
allowing $P$. gingivalis subvert immune surveillance contributing to increased virulence and periodontal pathogenesis. [85,87,129].

\section{Tobacco Smoke, $P$. gingivalis, and Periodontal Disease}

Our main objective in this thesis was to identify potential genomic instability and genetic rearrangements and alterations induced by cigarette smoke extract (CSE) in the periodontal and systemic pathogen Porphyromonas gingivalis, which is a keystone pathogen and strongly implicated in periodontal disease pathogenesis $[112,113,116,120,123]$. CSE is an environmental stressor to $P$. gingivalis and upon exposure, up-regulated $58 P$. gingivalis genes [115]. Of these, groups of genes encoding proteins involved in DNA translocation, replication and repair such as ruvC (PG1324), a putative integrase (PG0817), and radC (PG0894), as well as members of the tra family of proteins, traE (PG1483), traF (PG1482), and traK (PG1478) were up-regulated, which may indicate genomic instability and genetic alterations and repairs upon CSE exposure. Of particular interest to us was the up-regulation of insertion sequences (IS) ISPg3 (PG0299), ISPg5 (PG0943), and ISPg6 (PG1061).

Insertion sequences are relatively short sequences of DNA between 800 and 2,500 base pairs long and can greatly contribute to genomic variability and evolution. IS elements contain at least one open reading frame, which encodes a transposase enzyme that allows the IS element to move throughout the genome in either a cut-and-paste or copy-and-paste fashion. 


\section{Study Hypothesis}

Our main hypothesis is that exposure of cigarette smoke (CSE) would induce mutations and allelic variance in $P$. gingivalis. If so, we would establish that tobacco smoke may promote bacterial evolution with the potential for the emergence of more virulent clinical strains which may infect smokers and non-smokers alike. 


\section{CHAPTER TWO: MATERIALS AND METHODS}

\section{Materials}

P. gingivalis W83 was purchased from the American Type Culture Collection (Manassas, VA); P. gingivalis growth medium - Gifu Anaerobic Medium (GAM), was bought from Nissui Pharmaceutical (Tokyo, Japan). 3R4F standard reference cigarettes were obtained from the Kentucky Tobacco Research and Development Center (Lexington, KY). ). The Wizard genomic DNA purification kit came from Promega (Madison, WI). Primers were ordered from Bio-Synthesis, Inc. (Lewisville, TX).

\section{Bacterial Culture and In Vitro Modeling of Tobacco Exposure}

Porphyromonas gingivalis W83 was maintained as frozen stock culture. $P$. gingivalis was then grown in GAM or GAM conditioned media with cigarette smoke extract (GAM-CSE) under anaerobic conditions $\left(80 \% \mathrm{~N}_{2}, 10 \% \mathrm{H}_{2}, 10 \% \mathrm{CO}_{2}\right)$ at $37^{\circ} \mathrm{C}$ in a Coy Laboratories (Grass Lake Charter Township, MI) anaerobic chamber. P. gingivalis cells were harvested at mid- to late-exponential phase $\left(\mathrm{OD}_{600}=1.0\right.$, corresponding to $10^{9}$ cells per ml). GAM-CSE media was prepared using 3R4F reference cigarettes. Cigarette smoke was drawn through $50 \mathrm{~mL}$ of GAM using a syringe, and a three-way stopcock, in 
$35 \mathrm{~mL}$ drags every 20 seconds as described previously [115]. One cigarette was smoked per $50 \mathrm{~mL}$ of media. The CSE-conditioned medium was filtered $(0.22 \mathrm{~mm})$ and diluted to a concentration of $4000 \mathrm{ng} \mathrm{ml}$ nicotine. Nicotine content was determined by gas-liquid chromatography as described previously [133], and adjusted to $\mathrm{pH}$ 7.2. Upon reaching an $\mathrm{OD}_{600}$ of 1.0, P. gingivalis was sub-cultured into fresh GAM-CSE until 50 passages were completed.

\section{Isolation of Genomic DNA}

Genomic DNA was isolated from $1.5 \mathrm{ml}$ bacterial culture $\left(\mathrm{OD}_{600}=1.0\right.$, approximately $10^{9}$ cells $/ \mathrm{ml}$ ) after 50 passages in GAM-CSE with the Wizard genomic DNA purification kit according to manufacturer's instructions.

\section{Whole Genome Sequencing}

The complete genomic sequences of both control and CSE-exposed $P$. gingivalis (after 50 passages in GAM or GAM-CSE) were obtained using the Illumina HiSeq2000 sequencing platform with paired end sequencing and a read length of 100 base pairs. The paired end reads from the control and CSE treated samples were mapped, respectively, using the BWA program ([http://bio-bwa.sourceforge.net/] version 0.6 .1 ) to the $P$.

$\begin{array}{llll}\text { gingivalis } & \text { W83 } & \text { assembly } & \text { NC_002950.2 }\end{array}$ (http://www.ncbi.nlm.nih.gov/nuccore/NC_002950.2). The mapping process consisted first of running BWA program on each fastq file, followed by a run of sampe to create a SAM file from the R1 and R2 read pairs. The resulting SAM file was converted to a 
BAM file and sorted using Samtools ([http://samtools.sourceforge.net/] version 0.1.18). PCR duplicates were identified in each BAM file and removed using Picard Tools ([http://picard.sourceforge.net/] version 1.65) Mark Duplicates. Insertion and deletion (Indel) targets were identified using the Genome Analysis Toolkit (GATK) ([http://www.broadinstitute.org/gatk/] version 1.5) Realigner Target Creator. These targets were then fed into GATK Indel Realigner. These cleaned reads were then run through Samtools mpileup to create pileup files, which were used to derive absolute allele counts at each position for the respective BAM files. The de-duplicated, realigned BAM files were loaded into the Intrepid Bioinformatics data management system, and may be viewed in their entirety at:

\section{Control:}

http://server1.intrepidbio.com/FeatureBrowser/ngsdatasetrecord/record?ngsrecord=64411 $\underline{08798}$

\section{CSE:}

http://server1.intrepidbio.com/FeatureBrowser/ngsdatasetrecord/record?ngsrecord=64411 $\underline{08803}$

\section{Confirmation of Point Mutations}

Confirmation (i) - Visual Inspection

Each statistically significant identified mutation was viewed in Integrative Genomics Viewer (IGV) [intrepidbio.com] to view the position of the mutations within 
the read as well as the read lengths to determine possible sequencing errors and misalignments.

Confirmation (ii) - BLASTn

A nucleotide BLAST search [http://blast.ncbi.nlm.nih.gov/] was also conducted at for each significant mutation read sequence to control for alignment errors.

\section{Confirmation (iii) - Allele Specific PCR}

The presence of the variant alleles were also confirmed using allele specific PCR, when possible. PCR reactions were performed in triplicates as described by Calero et al. [136] using 50ng of template DNA, $0.3 \mu \mathrm{M}$ of each Primer and $12.5 \mu \mathrm{l}$ of $\mathrm{RT}^{2} \mathrm{SYBR}$ Green ROX Mastermix on an Applied Biosystems 7500 Real-Time PCR System (Invitrogen, Carlsbad, CA) under following conditions: $2 \mathrm{~min}$ at $50^{\circ} \mathrm{C}, 10 \mathrm{~min}$ at $95^{\circ} \mathrm{C}, 40$ cycles of $15 \mathrm{sec}$ at $95^{\circ} \mathrm{C}$ and $1 \mathrm{~min}$ at $55^{\circ} \mathrm{C}$, with data collection during the $55^{\circ} \mathrm{C}$ step. A dissociation stage followed each run to allow for the analysis of melting curves. Changes in cycle threshold $(\mathrm{Ct})$ values were calculated by subtracting the mean $\mathrm{Ct}$ value of the variant alleles from the mean $\mathrm{Ct}$ value of the control alleles $\left(\mathrm{Ct}_{\text {variant }}-\mathrm{Ct}_{\text {control }}=\Delta \mathrm{Ct}\right)$.

\section{Statistical Analyses}

\section{$\underline{\text { Mutations }}$}

Sequence variants were screened for statistical analysis using the genome analysis toolkit (GATK), to identify sequence variants in the CSE and control population samples. 
Allele and insertion / deletion counts from these 190 sequence variants were analyzed for differences in allele or insertion / deletion frequency between control and CSE samples using chi-squared tests of the $2 \times 2$ table consisting of the two most common alleles or the most common allele along with insertion / deletion count. Corresponding p-values were adjusted for multiple comparisons using the Benjamini-Hochberg approach [134], which controls the false-discovery rate.

\section{Allele frequency/Read Variance}

The mean- and median- percent variation from the reference allele (http://www.ncbi.nlm.nih.gov/nuccore/NC_002950.2) (referred to as the 'minor allele frequency') was calculated across all nucleotides separately for both CSE and control samples genomes. Simulated allele counts were generated at each position using an averaged minor allele frequency between CSE and control samples. Each nucleotide was treated as independent. Minor allele frequencies were then averaged across the genome for each of these simulated CSE and control genome counts. This was repeated 1000 times, and then this distribution of differences between CSE and control compared to the true differences in both mean and median minor allele frequencies. An empirical p-value was determined by the proportion of values from the null distribution that are greater than the observed (original) difference. 


\section{CHAPTER THREE: CSE EXPOSURE PROMOTES GENOMIC INSTABILITY AND BACTERIAL EVOLUTION.}

\section{Results}

CSE Induces Point Mutations in P. gingivalis

Complete genomic sequences of control and CSE-exposed $P$. gingivalis (after 50 passages in GAM-CSE) were obtained using the Illumina HiSeq2000 sequencing platform, using paired end sequencing with reads approximately 100 base-pairs long. It is important to acknowledge that the final whole genome sequence for this experiment is representative of a composite genome of all CSE-induced alterations to $P$. gingivalis extant after 50 passages. Considering, after prolonged passaging in CSE, there were likely to be multiple mutations induced in individual replicating bacterial cells. Naturally, many of these mutations will have been further propagated. Therefore, the final microbial population is likely to be composed of a variety of chromosomal sequences and should not be considered a single entity.

Keeping this in mind, CSE proved to be an environmental stressor capable of inducing $22(\mathrm{p}<0.05)$ point mutations as presented in Table 1: 


\section{TABLE 1: Statistically Significant Mutations}

\begin{tabular}{|l|l|}
\hline Position & p-value \\
\hline 2282335 & 0 \\
\hline 2068758 & $7.38 \mathrm{E}-99$ \\
\hline 1463982 & $1.04 \mathrm{E}-92$ \\
\hline 2307777 & $1.06 \mathrm{E}-79$ \\
\hline 1707468 & $3.76 \mathrm{E}-42$ \\
\hline 2068683 & $5.04 \mathrm{E}-41$ \\
\hline 2068587 & $1.63 \mathrm{E}-32$ \\
\hline 1371743 & $1.00 \mathrm{E}-23$ \\
\hline 2068514 & $4.78 \mathrm{E}-16$ \\
\hline 275989 & $1.99 \mathrm{E}-15$ \\
\hline 1963511 & $3.95 \mathrm{E}-10$ \\
\hline 2068717 & $5.81 \mathrm{E}-10$ \\
\hline 2068645 & $3.24 \mathrm{E}-09$ \\
\hline 2068720 & $1.26 \mathrm{E}-08$ \\
\hline 1963452 & $2.29 \mathrm{E}-06$ \\
\hline 1963451 & $4.39 \mathrm{E}-06$ \\
\hline 2068718 & 0.001 \\
\hline 1963860 & 0.001 \\
\hline 1963868 & 0.008 \\
\hline 599501 & 0.014 \\
\hline 2068540 & 0.017 \\
\hline 2049674 & 0.034 \\
\hline
\end{tabular}

\section{Confirmation of Point Mutations}

We were able to confirm 17/22 (77\%) mutations from Table 1 using: (i) - visual inspection; (ii) - BLASTn; and (iii) - allele-specific PCR [described previously in Methods]. Due to several variants being located within highly repetitive DNA sequence patterns - which does not allow for effective primer design - allele-specific PCR was only employed for three positions as shown in Table 2 and Table 3. 
Table 2: Primer design for allele-specific PCR

\begin{tabular}{|c|c|c|}
\hline Position & $\begin{array}{l}\text { Sequence Forward Primer } \\
\left(5^{\prime}-3^{\prime}\right)\end{array}$ & Sequence Reverse Primer (5'-3') \\
\hline 1707468 & CATGCT CATCGGTATTCTTG & $\begin{array}{l}\text { TATTTTTCGTGGGGCAAGATG } \\
\text { CTCCCTATCGTATCTCTCTAG } \\
\text { (Deletion) }\end{array}$ \\
\hline 2282335 & TATGCTGCTTTCTACTGCCCA & $\begin{array}{l}\text { GTGAGGAACGCCACGCCT } \\
\text { GTGAGGAACGCCACGCCC } \\
\text { (C Allele) }\end{array}$ \\
\hline 2307777 & CTATCCTGACTTTGTCGTCGG & $\begin{array}{l}\text { AGTCGAAGAGCTGCTTTTTTTC } \\
\text { AGTCGAAGAGCTGCTTTTTTC } \\
\text { (Deletion) }\end{array}$ \\
\hline
\end{tabular}

Table 3: Allele-specific real-time PCR $\Delta C t$ Values

\begin{tabular}{|l|c|c|c|c|c|c|}
\hline & \multicolumn{2}{|c|}{ GAM control culture } & \multicolumn{3}{c|}{$\mathbf{5 0}^{\text {th }}$ passage in CSE } \\
\hline Position & $\begin{array}{c}\text { Ct Regular } \\
\text { Allele }\end{array}$ & $\begin{array}{c}\text { Ct Variant } \\
\text { Allele }\end{array}$ & $\Delta \mathrm{Ct}$ & $\begin{array}{c}\text { Ct Regular } \\
\text { Allele }\end{array}$ & $\begin{array}{c}\text { Ct Variant } \\
\text { Allele }\end{array}$ & $\Delta \mathrm{Ct}$ \\
\hline $\mathbf{1 7 0 7 4 6 8}$ & 11.91 & 24.60 & 12.69 & 12.65 & 12.26 & -0.39 \\
\hline $\mathbf{2 2 8 2 3 3 5}$ & 11.89 & 14.72 & 2.83 & 15.83 & 11.24 & -4.59 \\
\hline $\mathbf{2 3 0 7 7 7 7}$ & 17.08 & 16.10 & -0.98 & 18.15 & 14.45 & -3.7 \\
\hline
\end{tabular}

The negative CSE $\Delta \mathrm{Ct}$ values in Table 3 represent a higher amount of variant allele present than the reference allele and control culteres as given by, (Ctvariant Ctcontrol $=\Delta \mathrm{Ct}$ ). values The 17 mutations spanned across the entire genome and were comprised of four deletions, three insertions, and ten Single Nucleotide Polymorphisms (SNPs) accounting for 18\%, 23\%, and 59\%, respectively as shown in Tables 4-6 below.

TABLE 4: Deletions

\begin{tabular}{|l|l|l|l|}
\hline Position & Gene & Function & Deletion \\
\hline $\mathbf{2 3 0 7 7 7 7}$ & PG2200 & hypothetical protein & T \\
\hline $\mathbf{1 7 0 7 4 6 8}$ & IGR & -- & $\begin{array}{l}\text { ATCTTGCCCCACGAAAAATAGA } \\
\text { TTAAACTCATTCTCGGAACGCA } \\
\text { CTCTAAATCAA }\end{array}$ \\
\hline $\mathbf{2 0 6 8 5 8 7}$ & IGR & -- & TACGGAC \\
\hline $\mathbf{2 0 6 8 5 1 4}$ & IGR & -- & TACGGAC \\
\hline
\end{tabular}


TABLE 5: Insertions

\begin{tabular}{|l|l|l|l|}
\hline Position & Gene & Function & Insertion \\
\hline $\mathbf{2 0 6 8 7 1 7}$ & IGR & -- & G \\
\hline $\mathbf{2 0 6 8 6 4 5}$ & IGR & -- & AGTAAT \\
\hline $\mathbf{2 0 6 8 5 4 0}$ & IGR & -- & CAG \\
\hline
\end{tabular}

TABLE 6: Single Nucleotide Polymorphisms (SNPs)

\begin{tabular}{|l|l|l|l|l|}
\hline Position & Gene & Function & Control & Mutation \\
\hline $\mathbf{2 2 8 2 3 3 5}$ & PG2174 & hypothetical protein & T & C \\
\hline $\mathbf{2 0 6 8 7 5 8}$ & IGR & -- & T & A \\
\hline $\mathbf{2 0 6 8 6 8 3}$ & IGR & -- & A & C \\
\hline $\mathbf{1 9 6 3 5 1 1}$ & PG1864 & leucine-rich protein & A & T \\
\hline $\mathbf{2 0 6 8 7 2 0}$ & IGR & -- & A & T \\
\hline $\mathbf{1 9 6 3 4 5 2}$ & PG1864 & leucine-rich protein & T & A \\
\hline $\mathbf{1 9 6 3 4 5 1}$ & PG1864 & leucine-rich protein & G & T \\
\hline $\mathbf{2 0 6 8 7 1 8}$ & IGR & -- & C & A \\
\hline $\mathbf{1 9 6 3 8 6 0}$ & PG1864 & leucine-rich protein & T & C \\
\hline $\mathbf{1 9 6 3 8 6 8}$ & PG1864 & leucine-rich protein & T & G \\
\hline
\end{tabular}

Five of the seven gene-based point mutations $(71 \%)$ were SNPs located within PG1864, a leucine-rich protein. The other two gene-based mutations were located individually within PG2174 (SNP) and PG2200 (Deletion) both of which are hypothetical proteins.

CSE-exposure increases minor allele frequency (read variance) in $P$. gingivalis

The mean- and median- percent variation from the major allele was calculated across all nucleotides separately for both CSE and control samples genomes. Simulated allele counts were generated at each position using an averaged minor allele frequency between CSE and control samples. Each nucleotide was treated as independent. Minor allele frequencies were then averaged across the genome for each of these simulated CSE and control genome counts. This was repeated 1000 times, and then this distribution of 
differences between CSE and control compared to the true differences in both mean and median minor allele frequencies. The results are as shown in Table 7 below as proportions, rather than percentages.

TABLE 7: WGS Read Allele Variance

\begin{tabular}{|l|l|l|}
\hline & Mean & Median \\
\hline Control & 0.00266653 & 0.00147384 \\
\hline CSE & 0.00271093 & 0.00149813 \\
\hline
\end{tabular}

CSE samples had slightly higher mean and median variance than control samples, which was determined to be significant $(p<0.001)$ in comparison to control samples.

\section{Discussion}

Cigarette smoking increases vulnerability to $P$. gingivalis infection and increases susceptibility to periodontitis, but reduces clinical signs of overt inflammation. The underlying mechanisms involved in this clinical conundrum are still being investigated and remain to be determined. However, it has been hypothesized that cigarette smoke suppresses host immunity while in contributing to increased bacterial virulence [17]. Several studies have already implicated tobacco smoke in increasing the virulence of $P$. gingivalis. We know from previous data that genetic expression of $P$. gingivalis is differentially regulated upon exposure to CSE. Major fimbrial genes (PG2133, PG2134) were induced, while several genes associated with capsular biosynthesis $(c a p K$, wecC, PG0117, PG0118) were suppressed [115,126]. Evidence also suggests tobacco smoke has the ability to induce alterations to the Lipid A region of $P$. gingivalis LPS reducing inflammatory potential, therefore increasing virulence and promoting proliferation [127]. 
It has also been shown that tobacco smoke contains several toxic chemicals as well as the capacity to generate reactive oxygen species [5-7] which have damaging effects on bacterial DNA [90-92]. The previous microarray study [115] also indicated the up-regulation of several genes encoding proteins involved in DNA replication, translocation, and repair.

Our experimental results show that $P$. gingivalis exposure to CSE promotes genomic instability by inducing 17 point mutations as well as inducing statistically significant $(\mathrm{p}<0.001)$ read variance. After only 50 passages of exposure over a $23.5 \mathrm{Mb}$ genome, our results imply considerable potential for genetic variance in $P$. gingivalis induced by CSE, which is in line with our hypothesis.

Given the relatively small number of significant point mutations across the entire P. gingivalis genome, striking clusters were noted in certain regions. For example, nine of the observed variants occur in the region between 2068514 and 2068758 (IGR-[2]**), while five occurred in the region between 1963451 and 1963868 (PG1864*) as shown in Figure 2 [135] below. 


\section{Figure 2: 17 CSE-induced mutations throughout the $P$. gingivalis genome}
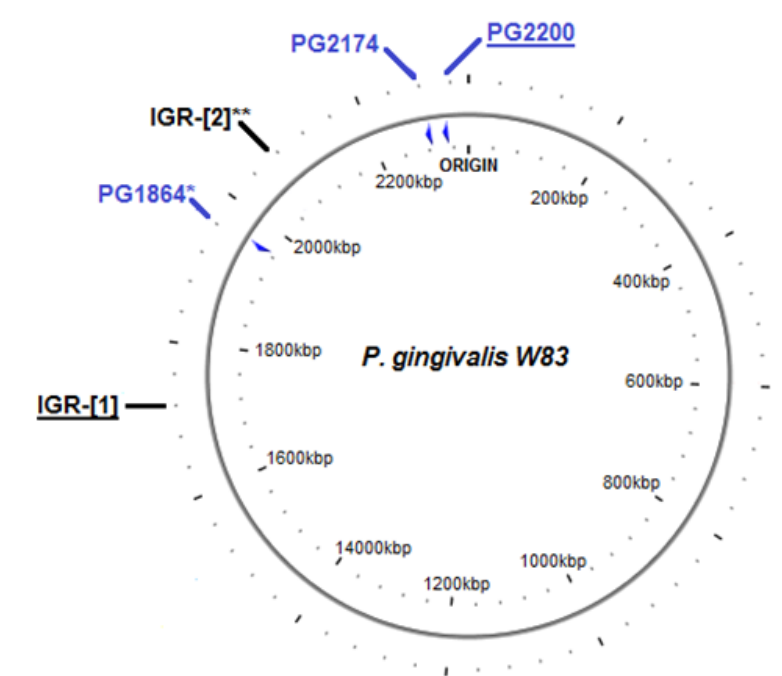

- Gene - Reverse Strand

- Intergenetic Region

$\underline{P G(x x x x)}$ - Indicates Indel

$P G(x x x x)$ - Indicates Mutation

* - Multiple Positions

** - Multiple Positions/ $>1$ Mutation Type

\begin{tabular}{|c|c|c|}
\hline Figure Position & Genomic Position(s) & \multirow{6}{*}{$\begin{array}{l}\text { (Position)-Confirmed using Visual and BLAST } \\
\text { confirmations methods. } \\
\text { (Position)-Confirmed using allele-specific PCR } \\
\text { in addition to Visual and BLAST confirmations. }\end{array}$} \\
\hline IGR-[1] & 1707468 & \\
\hline$\overline{\text { IGR-[2] }} * *$ & $\begin{array}{l}2068514,2068540,2068587,2068645,2068683,2068717, \\
2068718,2068720,2068758\end{array}$ & \\
\hline PG1864* & $1963511,1963452,1963451,1963860,1963868$ & \\
\hline PG2174 & 2282335 & \\
\hline PG2200 & 2307777 & \\
\hline
\end{tabular}

As for the limitations of the whole genome sequencing used in this project

(Illumina HiSeq2000), the short read technology (read length of approximately 100 bases) isn't optimal for characterizing regions with tandem repeats. Complementary, longer read technologies such as the ionTorrent, ionProton, or better still, PacBio will remove much of the ambiguity, and as a result will significantly improve our ability to characterize these regions.

We also did not explore whether CSE exposure additionally causes epigenetic changes, altering the activity of DNA by chemical modifications of DNA or histone proteins, for example by affecting methyl or acetyl adducts. Such information would provide additional valuable information and warrants future investigation to clarify the influence of cigarette smoke on the epigenetic level. 
In summary, smokers are more prone to infection with $\mathrm{P}$. gingivalis and to develop periodontitis, yet exhibit reduced clinical inflammation. Our experimental results gives further evidence to that cigarette smoke is an important driving force for bacterial evolution. We have found that Porphyromonas gingivalis' response to CSE exposure results in genome wide allele variance and point mutations. These results may explain in part the effects tobacco smoke in altering the virulence of this key periodontal pathogen that may contribute to the emergence of more virulent strains, which may infect smokers and non-smokers alike. 


\section{REFERENCES}

1. Smoking-attributable mortality, years of potential life lost, and productivity losses--United States, 2000-2004. MMWR Morb Mortal Wkly Rep, 2008. 57(45): p. 1226-8.

2. Brown, L., B. Johns, and T. Wall, The economics of periodontal diseases. Periodontol 2000, 2002. 29: p. 223-34.

3. Jha, P., F. J. Chaloupka, M. Corrao, and B. Jacob. 2006. Reducing the burden of smoking world-wide: effectiveness of interventions and their coverage. Drug Alcohol Rev 25:597-609.

4. Petersen, P.E., Tobacco and oral health--the role of the world health organization. Oral Health Prev Dent, 2003. 1(4): p. 309-15.

5. Mehta, H., K. Nazzal, and R.T. Sadikot, Cigarette smoking and innate immunity. Inflamm Res, 2008. 57(11): p. 497-503.

6. Stampfli, M. and G. Anderson, How cigarette smoke skews immune responses to promote infection, lung disease and cancer. Nat Rev Immunol, 2009. 9(5): p. 377-84.

7. Gilmour, M. I., M. S. Jaakkola, S. J. London, A. E. Nel, and C. A. Rogers. 2006. How exposure to tobacco smoke, outdoor air pollutants, and increased pollen burdens influences the incidence of asthma. Environ Health Perspect 114:627633.

8. Jha, P., Avoidable global cancer deaths and total deaths from smoking. Nat Rev Cancer, 2009. 9(9): p. 655-64.

9. Salmasi, G., et al., Environmental tobacco smoke exposure and perinatal outcomes: a systematic review and meta-analyses. Acta Obstet Gynecol Scand, 2010. 89(4): p. 423-41.

10. Kabir, M.A., K.L. Goh, and M.M. Khan, Adolescent Tobacco Use and Its Determinants: Evidence From Global Youth Tobacco Survey, Bangladesh 2007. Asia Pac J Public Health, 2013.

11. Vardavas, C.I. and D.B. Panagiotakos, The causal relationship between passive smoking and inflammation on the development of cardiovascular disease: a review of the evidence. Inflamm Allergy Drug Targets, 2009. 8(5): p. 328-33.

12. El-Mohandes, A.A., et al., An intervention to reduce environmental tobacco smoke exposure improves pregnancy outcomes. Pediatrics, 2010. 125(4): p. 721-8.

13. Wolf, P.A., et al., Cigarette smoking as a risk factor for stroke. The Framingham Study. JAMA, 1988. 259(7): p. 1025-9.

14. Csordas, A. and D. Bernhard, The biology behind the atherothrombotic effects of cigarette smoke. Nat Rev Cardiol, 2013.

15. Schroeter, M.R., et al., Cigarette smoke exposure promotes arterial thrombosis and vessel remodeling after vascular injury in apolipoprotein E-deficient mice. $\mathrm{J}$ 
Vasc Res, 2008. 45(6): p. 480-92.

16. Patel, B.P., et al., Mutagen sensitivity in oral cancer patients, healthy tobacco chewers and controls. Acta Cytol, 2010. 54(2): p. 169-74.

17. Bagaitkar, J., D.R. Demuth, and D.A. Scott, Tobacco use increases susceptibility to bacterial infection. Tob Induc Dis, 2008. 4: p. 12.

18. Ezzati, M., et al., Role of smoking in global and regional cardiovascular mortality. Circulation, 2005. 112(4): p. 489-97.

19. Mercado, C. and E.A. Jaimes, Cigarette smoking as a risk factor for atherosclerosis and renal disease: novel pathogenic insights. Curr Hypertens Rep, 2007. 9(1): p. 66-72.

20. Yadav, D. and D.C. Whitcomb, The role of alcohol and smoking in pancreatitis. Nat Rev Gastroenterol Hepatol, 2010. 7(3): p. 131-45

21. Martins Junior, E.V., et al., [Smoking and inflammatory bowel disease: an epidemiological case-control study]. Arq Gastroenterol, 1996. 33(2): p. 74-8.

22. Smyk, D.S., et al., Smoking as a risk factor for autoimmune liver disease: what we can learn from primary biliary cirrhosis. Ann Hepatol, 2012. 11(1): p. 7-14.

23. Baelum, V. and R. Lopez, Defining and classifying periodontitis: need for a paradigm shift? Eur J Oral Sci, 2003. 111(1): p. 2-6.

24. Pihlstrom, B.L., B.S. Michalowicz, and N.W. Johnson, Periodontal diseases. Lancet, 2005. 366(9499): p. 1809-20.

25. Huttunen, R., T. Heikkinen, and J. Syrjanen, Smoking and the outcome of infection. J Intern Med, 2011. 269(3): p. 258-69.

26. Deshpande, A., Epidemiology of community acquired pneumonia. J Assoc Physicians India, 2012. 60 Suppl: p. 6.

27. Armitage, G.C., Clinical evaluation of periodontal diseases. Periodontol 2000, 1995. 7: p. 39-53.

28. Sopori, M., Effects of cigarette smoke on the immune system. Nat Rev Immunol, 2002. 2(5): p. 372-7.

29. Borrell, L.N. and P.N. Papapanou, Analytical epidemiology of periodontitis. J Clin Periodontol, 2005. 32 Suppl 6: p. 132-58.

30. Jha, P., et al., 21st-century hazards of smoking and benefits of cessation in the United States. N Engl J Med, 2013. 368(4): p. 341-50.

31. Kinane, D.F., et al., Human variability in innate immunity. Periodontol 2000, 2007. 45: p. 14-34.

32. Calsina, G., J.M. Ramon, and J.J. Echeverria, Effects of smoking on periodontal tissues. J Clin Periodontol, 2002. 29(8): p. 771-6.

33. Airila-Mansson, S., B. Soder, and B. Klinge, Bone height changes in individuals with periodontal disease: a 17-year prospective longitudinal study. J Clin Periodontol, 2005. 32(7): p. 822-7.

34. Eke, P.I., et al., Prevalence of periodontitis in adults in the United States: 2009 and 2010. J Dent Res, 2012. 91(10): p. 914-20.

35. Bergstrom, J., S. Eliasson, and J. Dock, Exposure to tobacco smoking and periodontal health. J Clin Periodontol, 2000. 27(1): p. 61-8.

36. Palmer, R.M., et al., Mechanisms of action of environmental factors--tobacco smoking. J Clin Periodontol, 2005. 32 Suppl 6: p. 180-95.

37. Graves, D., Cytokines that promote periodontal tissue destruction. J Periodontol, 
2008. 79(8 Suppl): p. 1585-91.

38. Darveau, R.P., A. Tanner, and R.C. Page, The microbial challenge in periodontitis. Periodontol 2000, 1997. 14: p. 12-32.

39. James, J.A., et al., Effects of tobacco products on the attachment and growth of periodontal ligament fibroblasts. J Periodontol, 1999. 70(5): p. 518-25.

40. Brusselle, G.G., G.F. Joos, and K.R. Bracke, New insights into the immunology of chronic obstructive pulmonary disease. Lancet, 2011. 378(9795): p. 1015-26.

41. Rota, M.T., et al., Tobacco smoke in the development and therapy of periodontal disease: progress and questions. Bull Group Int Rech Sci Stomatol Odontol, 1999. 41(4): p. 116-22.

42. Bullen, C., Impact of tobacco smoking and smoking cessation on cardiovascular risk and disease. Expert Rev Cardiovasc Ther, 2008. 6(6): p. 883-95.

43. Onozaki, K., Etiological and biological aspects of cigarette smoking in rheumatoid arthritis. Inflamm Allergy Drug Targets, 2009. 8(5): p. 364-8.

44. Costenbader, K.H. and E.W. Karlson, Cigarette smoking and autoimmune disease: what can we learn from epidemiology? Lupus, 2006. 15(11): p. 737-45.

45. Nuorti, J.P., et al., Cigarette smoking and invasive pneumococcal disease. Active Bacterial Core Surveillance Team. N Engl J Med, 2000. 342(10): p. 681-9.

46. Akishima, S., et al., Cigarette-smoke-induced vasoconstriction of peripheral arteries: evaluation by synchrotron radiation microangiography. Circ J, 2007. 71(3): p. 418-22.

47. Holloway, R.A. and L.E. Donnelly, Immunopathogenesis of chronic obstructive pulmonary disease. Curr Opin Pulm Med, 2013.

48. Michael Pittilo, R., Cigarette smoking, endothelial injury and cardiovascular disease. Int J Exp Pathol, 2000. 81(4): p. 219-30.

49. Lehr, H.A., Microcirculatory dysfunction induced by cigarette smoking. Microcirculation, 2000. 7(6 Pt 1): p. 367-84.

50. Bostanci, N. and G.N. Belibasakis, Porphyromonas gingivalis: an invasive and evasive opportunistic oral pathogen. FEMS Microbiol Lett, 2012. 333(1): p. 1-9.

51. Peyyala, R., et al., Oral Epithelial Cell Responses to Multispecies Microbial Biofilms. J Dent Res, 2013.

52. Giannopoulou, C., A. Geinoz, and G. Cimasoni, Effects of nicotine on periodontal ligament fibroblasts in vitro. J Clin Periodontol, 1999. 26(1): p. 49-55.

53. Chang, Y.C., et al., Adverse effects of arecoline and nicotine on human periodontal ligament fibroblasts in vitro. J Clin Periodontol, 2001. 28(3): p. 27782.

54. Katono, T., et al., Nicotine treatment induces expression of matrix metalloproteinases in human osteoblastic Saos-2 cells. Acta Biochim Biophys Sin (Shanghai), 2006. 38(12): p. 874-82.

55. Chang, Y.C., et al., Induction of c-fos expression by nicotine in human periodontal ligament fibroblasts is related to cellular thiol levels. J Periodontal Res, 2003. 38(1): p. 44-50.

56. Scott, D.A. and D.L. Singer, Suppression of overt gingival inflammation in tobacco smokers - clinical and mechanistic considerations. Int J Dent Hyg, 2004. 2(3): p. 104-10.

57. Scott, D.A. and R.M. Palmer, The influence of tobacco smoking on adhesion 
molecule profiles. Tob Induc Dis, 2002. 1(1): p. 7-25.

58. Rezavandi, K., et al., Expression of ICAM-1 and E-selectin in gingival tissues of smokers and non-smokers with periodontitis. J Oral Pathol Med, 2002. 31(1): p. 59-64.

59. Singh, A., et al., The capsule of Porphyromonas gingivalis leads to a reduction in the host inflammatory response, evasion of phagocytosis, and increase in virulence. Infect Immun, 2011. 79(11): p. 4533-42.

60. Aduse-Opoku, J., et al., Identification and characterization of the capsular polysaccharide (K-antigen) locus of Porphyromonas gingivalis. Infect Immun, 2006. 74(1): p. 449-60.

61. Brunner, J., et al., The capsule of Porphyromonas gingivalis reduces the immune response of human gingival fibroblasts. BMC Microbiol, 2010. 10: p. 5.

62. Yilmaz, Ö., Yao, L., Maeda, K., Rose, T. M., Lewis, E. L., Duman, M., Lamont, R. J. and Ojcius, D. M. (2008), ATP scavenging by the intracellular pathogen Porphyromonas gingivalis inhibits $P 2 X_{7}$-mediated host-cell apoptosis. Cellular Microbiology, 10: 863-875. doi: 10.1111/j.1462-5822.2007.01089.x

63. Yilmaz, O., K. Watanabe, and R.J. Lamont, Involvement of integrins in fimbriaemediated binding and invasion by Porphyromonas gingivalis. Cell Microbiol, 2002. 4(5): p. 305-14.

64. Wang, M., et al., Fimbrial proteins of porphyromonas gingivalis mediate in vivo virulence and exploit TLR2 and complement receptor 3 to persist in macrophages. J Immunol, 2007. 179(4): p. 2349-58.

65. Paster, B.J., et al., The breadth of bacterial diversity in the human periodontal pocket and other oral sites. Periodontol 2000, 2006. 42: p. 80-7.

66. Hajishengallis, G. and R.J. Lamont, Beyond the red complex and into more complexity: the polymicrobial synergy and dysbiosis (PSD) model of periodontal disease etiology. Mol Oral Microbiol, 2012. 27(6): p. 409-19.

67. Belton, C.M., et al., Fluorescence image analysis of the association between Porphyromonas gingivalis and gingival epithelial cells. Cell Microbiol, 1999. 1(3): p. 215-23.

68. Laine, M.L., B.J. Appelmelk, and A.J. van Winkelhoff, Prevalence and distribution of six capsular serotypes of Porphyromonas gingivalis in periodontitis patients. J Dent Res, 1997. 76(12): p. 1840-4.

69. Potempa, J. and J. Travis, Porphyromonas gingivalis proteinases in periodontitis, a review. Acta Biochim Pol, 1996. 43(3): p. 455-65.

70. Chen, T., et al., Porphyromonas gingivalis gingipains and adhesion to epithelial cells. Infect Immun, 2001. 69(5): p. 3048-56.

71. Kitamura, Y., et al., Gingipains in the culture supernatant of Porphyromonas gingivalis cleave CD4 and CD8 on human T cells. J Periodontal Res, 2002. 37(6): p. 464-8.

72. Ozmeric, N., N.R. Preus, and I. Olsen, Genetic diversity of Porphyromonas gingivalis and its possible importance to pathogenicity. Acta Odontol Scand, 2000. 58(4): p. 183-7.

73. Amano, A., et al., Variations of Porphyromonas gingivalis fimbriae in relation to microbial pathogenesis. J Periodontal Res, 2004. 39(2): p. 136-42.

74. Lamont, R.J. and H.F. Jenkinson, Life below the gum line: pathogenic 
mechanisms of Porphyromonas gingivalis. Microbiol Mol Biol Rev, 1998. 62(4): p. 1244-63.

75. Inaba, H., et al., Heterogenic virulence and related factors among clinical isolates of Porphyromonas gingivalis with type II fimbriae. Oral Microbiol Immunol, 2008. 23(1): p. 29-35.

76. Hajishengallis, G., et al., Complement receptor 3 blockade promotes IL-12mediated clearance of Porphyromonas gingivalis and negates its virulence in vivo. J Immunol, 2007. 179(4): p. 2359-67.

77. Umemoto, T. and N. Hamada, Characterization of biologically active cell surface components of a periodontal pathogen. The roles of major and minor fimbriae of Porphyromonas gingivalis. J Periodontol, 2003. 74(1): p. 119-22.

78. Hasegawa, Y., et al., Anchoring and length regulation of Porphyromonas gingivalis Mfal fimbriae by the downstream gene product Mfa2. Microbiology, 2009. 155(Pt 10): p. 3333-47.

79. Khlgatian, M., et al., Fimbria-dependent activation of cell adhesion molecule expression in Porphyromonas gingivalis-infected endothelial cells. Infect Immun, 2002. 70(1): p. 257-67.

80. Bainbridge, B.W. and R.P. Darveau, Porphyromonas gingivalis lipopolysaccharide: an unusual pattern recognition receptor ligand for the innate host defense system. Acta Odontol Scand, 2001. 59(3): p. 131-8.

81. Wara-Aswapati, N., et al., Induction of Toll-Like Receptor Expression by Porphyromonas Gingivalis. J Periodontol, 2012.

82. Kadono, H., et al., Inhibition of osteoblastic cell differentiation by lipopolysaccharide extract from Porphyromonas gingivalis. Infect Immun, 1999. 67(6): p. 2841-6.

83. Liu, B., et al., Role of 38 mitogen-activated protein kinase pathway in Porphyromonas gingivalis lipopolysaccharide-induced VCAM-1 expression in human aortic endothelial cells. J Periodontol, 2012. 83(7): p. 955-62.

84. Liu, R., et al., P. gingivalis and E. coli lipopolysaccharides exhibit different systemic but similar local induction of inflammatory markers. J Periodontol, 2008. 79(7): p. 1241-7.

85. Darveau, R.P., et al., Porphyromonas gingivalis lipopolysaccharide contains multiple lipid A species that functionally interact with both toll-like receptors 2 and 4. Infect Immun, 2004. 72(9): p. 5041-51.

86. Hajishengallis, G., et al., Pathogen induction of CXCR4/TLR2 cross-talk impairs host defense function. Proc Natl Acad Sci U S A, 2008. 105(36): p. 13532-7.

87. Nemoto, E., et al., Regulation of cementoblast function by $P$. gingivalis lipopolysaccharide via TLR2. J Dent Res, 2006. 85(8): p. 733-8.

88. Dixon, D.R. and R.P. Darveau, Lipopolysaccharide heterogeneity: innate host responses to bacterial modification of lipid a structure. J Dent Res, 2005. 84(7): p. 584-95.

89. Al-Qutub, M.N., et al., Hemin-dependent modulation of the lipid A structure of Porphyromonas gingivalis lipopolysaccharide. Infect Immun, 2006. 74(8): p. 4474-85.

90. Aufderheide, M. and H. Gressmann, Mutagenicity of native cigarette mainstream smoke and its gas/vapour phase by use of different tester strains and cigarettes in 
a modified Ames assay. Mutat Res, 2008. 656(1-2): p. 82-7.

91. De Flora, S., et al., Bacterial mutagenicity of cigarette smoke and its interaction with ethanol. Mutagenesis, 1995. 10(1): p. 47-52.

92. Doolittle, D.J., et al., Comparative studies on the genotoxic activity of mainstream smoke condensate from cigarettes which burn or only heat tobacco. Environ Mol Mutagen, 1990. 15(2): p. 93-105.

93. Chen, X., et al., Cigarette smoking, salivary/gingival crevicular fluid cotinine and periodontal status. A 10-year longitudinal study. J Clin Periodontol, 2001. 28(4): p. 331-9.

94. Kallberg, H., et al., Smoking is a major preventable risk factor for rheumatoid arthritis: estimations of risks after various exposures to cigarette smoke. Ann Rheum Dis, 2011. 70(3): p. 508-11.

95. Carlens, C., et al., Smoking, use of moist snuff, and risk of chronic inflammatory diseases. Am J Respir Crit Care Med, 2010. 181(11): p. 1217-22.

96. Bernardi, L., et al., Autonomic and cerebrovascular abnormalities in mild COPD are worsened by chronic smoking. Eur Respir J, 2008. 32(6): p. 1458-65.

97. Gunsolley, J.C., et al., The effect of race, smoking and immunoglobulin allotypes on IgG subclass concentrations. J Periodontal Res, 1997. 32(4): p. 381-7.

98. Ginns, L.C., et al., T-lymphocyte subsets in smoking and lung cancer: Analysis of monoclonal antibodies and flow cytometry. Am Rev Respir Dis, 1982. 126(2): p. 265-9.

99. Hoser, G., et al., Flow cytometric evaluation of lymphocyte subpopulations in $B A L F$ of healthy smokers and nonsmokers. Folia Histochem Cytobiol, 1999. 37(1): p. 25-30.

100. Hoser, G., et al., Flow cytometric evaluation of lymphocyte subpopulations in BALF of healthy smokers and nonsmokers. Folia Histochem Cytobiol, 1999. 37(1): p. 25-30.

101. Richardson, M.A., Upper airway complications of cigarette smoking. J Allergy Clin Immunol, 1988. 81(5 Pt 2): p. 1032-5.

102. Wang XL, Scott DA: Molecular Mechanisms of Tobacco-Induced Diseases. 2006.

103. Dye, J.A. and K.B. Adler, Effects of cigarette smoke on epithelial cells of the respiratory tract. Thorax, 1994. 49(8): p. 825-34.

104. McMaster, S.K., et al., Cigarette smoke inhibits macrophage sensing of Gram-negative bacteria and lipopolysaccharide: relative roles of nicotine and oxidant stress. Br J Pharmacol, 2008. 153(3): p. 536-43.

105. Zappacosta, B., et al., Effect of cigarette smoke extract on the polymorphonuclear leukocytes chemiluminescence: influence of a filter containing glutathione. Luminescence, 2005. 20(2): p. 73-5.

106. Sorensen, L.T., et al., Effect of smoking and abstention on oxidative burst and reactivity of neutrophils and monocytes. Surgery, 2004. 136(5): p. 1047-53.

107. Robbins, C.S., et al., Cigarette smoke exposure impairs dendritic cell maturation and T cell proliferation in thoracic lymph nodes of mice. J Immunol, 2008. 180(10): p. 6623-8.

108. El Ahmer, O.R., et al., The effect of cigarette smoke on adherence of respiratory pathogens to buccal epithelial cells. FEMS Immunol Med Microbiol, 
1999. 23(1): p. 27-36.

109. Zonuz, A.T., et al., Effect of cigarette smoke exposure on the growth of Streptococcus mutans and Streptococcus sanguis: an in vitro study. Nicotine Tob Res, 2008. 10(1): p. 63-7.

110. Nelson, K.E., et al., Complete genome sequence of the oral pathogenic Bacterium porphyromonas gingivalis strain W83. J Bacteriol, 2003. 185(18): p. 5591-601.

111. Socransky, S.S., et al., Microbial complexes in subgingival plaque. J Clin Periodontol, 1998. 25(2): p. 134-44.

112. Darveau, R.P., G. Hajishengallis, and M.A. Curtis, Porphyromonas gingivalis as a potential community activist for disease. J Dent Res, 2012. 91(9): p. 816-20.

113. Igboin, C.O., A.L. Griffen, and E.J. Leys, Porphyromonas gingivalis strain diversity. J Clin Microbiol, 2009. 47(10): p. 3073-81.

114. Griffen, A.L., et al., Porphyromonas gingivalis strain variability and periodontitis. J Clin Microbiol, 1999. 37(12): p. 4028-33.

115. Bagaitkar, J., et al., Tobacco-induced alterations to Porphyromonas gingivalis-host interactions. Environ Microbiol, 2009. 11(5): p. 1242-53.

116. Kawada, M., et al., Prevalence of Porphyromonas gingivalis in relation to periodontal status assessed by real-time PCR. Oral Microbiol Immunol, 2004.

19(5): p. 289-92.

117. Honda, K., Porphyromonas gingivalis sinks teeth into the oral microbiota and periodontal disease. Cell Host Microbe, 2011. 10(5): p. 423-5.

118. Genco, C.A., T. Van Dyke, and S. Amar, Animal models for Porphyromonas gingivalis-mediated periodontal disease. Trends Microbiol, 1998. 6(11): p. 444-9.

119. Darveau, R.P., Periodontitis: a polymicrobial disruption of host homeostasis. Nat Rev Microbiol, 2010. 8(7): p. 481-90.

120. Genco, C.A., T. Van Dyke, and S. Amar, Animal models for Porphyromonas gingivalis-mediated periodontal disease. Trends Microbiol, 1998. 6(11): p. 444-9.

121. Hill, D.A. and D. Artis, Intestinal bacteria and the regulation of immune cell homeostasis. Annu Rev Immunol, 2010. 28: p. 623-67

122. Round, J.L. and S.K. Mazmanian, The gut microbiota shapes intestinal immune responses during health and disease. Nat Rev Immunol, 2009. 9(5): p. 313-23.

123. Haffajee, A.D., et al., The effect of SRP on the clinical and microbiological parameters of periodontal diseases. J Clin Periodontol, 1997. 24(5): p. 324-34.

124. Bagaitkar, J., et al., Tobacco smoke augments Porphyromonas gingivalisStreptococcus gordonii biofilm formation. PLoS One, 2011. 6(11): p. e27386.

125. Davey, M.E. and M.J. Duncan, Enhanced biofilm formation and loss of capsule synthesis: deletion of a putative glycosyltransferase in Porphyromonas gingivalis. J Bacteriol, 2006. 188(15): p. 5510-23.

126. Bagaitkar, J., et al., Tobacco upregulates $P$. gingivalis fimbrial proteins which induce TLR2 hyposensitivity. PLoS One, 2010. 5(5): p. e9323. 
127. Buduneli, N., et al., Fatty acid profiles in smokers with chronic periodontitis. J Dent Res, 2011.90(1): p. 47-52.

128. Dixon, D.R. and R.P. Darveau, Lipopolysaccharide heterogeneity: innate host responses to bacterial modification of lipid a structure. J Dent Res, 2005. 84(7): p. 584-95.

129. Herath, T.D., et al., Porphyromonas gingivalis lipopolysaccharide lipid $A$ heterogeneity differentially modulates the expression of IL-6 and IL-8 in human gingival fibroblasts. J Clin Periodontol, 2011. 38(8): p. 694-701.

130. Jin, L.J., et al., Global oral health inequalities: task group--periodontal disease. Adv Dent Res, 2011. 23(2): p. 221-6.

131. Huang da, W., B.T. Sherman, and R.A. Lempicki, Bioinformatics enrichment tools: paths toward the comprehensive functional analysis of large gene lists. Nucleic Acids Res, 2009. 37(1): p. 1-13.

132. Huang da, W., B.T. Sherman, and R.A. Lempicki, Systematic and integrative analysis of large gene lists using DAVID bioinformatics resources. Nat Protoc, 2009. 4(1): p. 44-57.

133. Fraser, H.S., et al., Elevated systemic concentrations of soluble ICAM-1 (sICAM) are not reflected in the gingival crevicular fluid of smokers with periodontitis. J Dent Res, 2001. 80(7): p. 1643-7.

134. Benjamini Y, Hochberg Y: Controlling the false discovery rate: a practical and powerful approach to multiple testing. J R Stat Soc 1995, 57:289-300.

135. Stothard P, Wishart DS. Circular genome visualization and exploration using CGView. Bioinformatics 21:537-539.

136. Calero O, Hortigüela R, Bullido MJ, Calero M. J Neurosci Methods. 2009 Oct 15;183(2):238-40. doi: 10.1016/j.jneumeth.2009.06.033. Epub 2009 Jul 5. 


\section{CURRICULUM VITAE}

\section{Education}

- The Ohio State University 2005-2007

- University of Kentucky 2008-2011

- University of Louisville 2011-Present
A.A.

B.S. Biology

M.S. Oral Biology Candidate

\section{Research Experience}

\section{Virology Study (Undergrad-UK)}

It was hypothesized that Human Herpes Virus (HHV) promotes and increases the risk of periodontal infections. Based on conflicting data, the goal of this research project was to study the potential role that HHVs play in periodontal disease using an experimental rat model.

To examine this question my mentor and I studied the influence of rat cytomegalovirus (RCMV), on the development of periodontitis using a rat model of periodontal disease. RCMV is genetically related to human cytomegalovirus, one of the most common types of herpes virus found at sites of periodontal infection. Our research investigated how the time-course of acquisition of RCMV infection influenced the 
progression of periodontal disease in rats colonized with a human periodontitis bacterial strain, Porphyromonas gingivalis.

Two experiments were designed to investigate potential interactions between these agents in promoting periodontitis in immunocompetent (Study-1) and immunosuppressed rats (Study-2). In these studies, 4-5 week-old rats were placed in and control and experimental treatment groups and inoculated with RCMV, P. gingivalis, or both agents at varying time-points. The RCMV was given by intraperitoneal injection and the $P$. gingivalis by oral gavage. Blood samples for determination of immune responses were collected at the start of the experiment and at approximate monthly intervals during the 4-month time-course of the experiment. At these times anesthetic was administered to allow for the collection of dental plaque samples to characterize the inflammatory profile of the rat periodontal tissues and to determine the persistence of $P$. gingivalis and/or RCMV in the oral cavity. At the end of each experiment the rats will be euthanatized by inhalation of $\mathrm{CO}_{2}$ gas. Gingival (periodontal) tissues will be excised for histological and molecular analysis and the mandibles and maxillary bones removed for radiographic evaluation of alveolar bone loss related to periodontal disease.

Rats in the second study, modeling periodontal disease in immunosuppressed hosts (e.g. cancer, AIDS, or organ transplant patients), were treated with whole body irradiation or immunosuppressive drugs to impair their immune system. The rats were divided into similar treatment groups and diagnostically sampled like rats in Study-1. 


\section{Telomere Study (Undergrad-UK)}

In this independent research project I hypothesized the function of spatially clustered telomere end genes of an Aspergillus nidulans (fungus) chromosome served a functionally common purpose based on preliminary data which, indicated they were involved with metabolic/growth function. To test this theory I used software programs and bioinformatics databases such as Broad Institute, BLAST, and Vector NTI to sequence the genes in question, compare them against phylotypically related species to try and determine a predicted function for each gene. From these sequences, primers for DNA polymerase reactions were designed to construct gene knockouts. The knockouts were then used to test the functionality of each of the 6 identified genes I was examining, independently and together to try and determine their individual function and a possible hypothesized common function. Effects of gene knockouts were measured morphologically compared to control $A$. nidulans colonies.

\section{Future Academic Plans}

After completing this Master's Degree, I will be attending the University of Kentucky College of Dentistry beginning the fall 2013 semester and graduate with the Doctor of Medical Dentistry (DMD) Class of 2017. 